\title{
HARMONIC MAPS AND CLASSICAL SURFACE THEORY IN MINKOWSKI 3-SPACE
}

BY

\author{
TILLA KLOTZ MILNOR
}

\begin{abstract}
Harmonic maps from a surface $S$ with nondegenerate prescribed and induced metrics are characterized, showing that holomorphic quadratic differentials play the same role for harmonic maps from a surface with indefinite prescribed metric as they do in the Riemannian case. Moreover, holomorphic quadratic differentials are shown to arise as naturally on surfaces of constant $H$ or $K$ in $M^{3}$ as on their counterparts in $E^{3}$. The connection between the sine-Gordon. sinh-Gordon and cosh-Gordon equations and harmonic maps is explained. Various local and global results are established for surfaces in $M^{3}$ with constant $H$, or constant $K \neq 0$. In particular, the Gauss map of a spacelike or timelike surface in $M^{3}$ is shown to be harmonic if and only if $H$ is constant. Also. $K$ is shown to assume values arbitrarily close to $H^{2}$ on any entire, spacelike surface in $M^{3}$ with constant $H$. except on a hyperbolic cylinder.
\end{abstract}

1. Introduction. If a surface with prescribed Riemannian metric is harmonically immersed in a $q$-dimensional Riemannian manifold, a particular quadratic differential associated with the mapping is holomorphic. (See [6].) In what seem to be quite different circumstances, on surfaces of constant mean curvature $H$ or Gauss curvature $K \neq 0$ in Euclidean 3-space $E^{3}$, a certain quadratic differential defined by the geometry of the situation is holomorphic. (See [12,13 and 20].)

These facts are related in a simple way, as shown in Theorems 9 and 12 below. Indeed, once metrics are properly chosen, the identity maps on surfaces with constant $H$ or $K \neq 0$ in $E^{3}$ are prototypes for harmonic maps from surfaces, in a sense made clear by Theorems A and B in $\S 5$.

For rather compelling reasons, one does not expect to find holomorphic quadratic differentials when studying analogous objects in the non-Riemannian case. Looking, for example, at a harmonic map from Minkowski 2-space, or at a timelike surface with constant $H$ in Minkowski 3-space $M^{3}$, the indefinite prescribed metrics fail to provide a complex analytic structure on the surface, and the defining partial differential equations (which are elliptic in the Riemannian case) are hyperbolic.

Nonetheless, we show in this paper that holomorphic quadratic diffeentials play the same role for harmonic maps from a surface with indefinite prescribed metric as they do for harmonic maps from a surface with Riemannian prescribed metric. Moreover, we show that holomorphic quadratic differentials arise just as naturally on spacelike or timelike surfaces with constant $H$ or $K \neq 0$ in $M^{3}$ as for their

Received by the editors July 13, 1981 and, in revised form, June 29, 1982.

1980 Mathematics Subject Classification. Primary 58E20, 53B25, 53B30; Secondary 53C42.

${ }^{1}$ Written while on sabbatical leave from Rutgers University at the University of California, Berkeley. 
counterparts in $E^{3}$. (What really matters is that the surfaces should sit in a pseudo-Riemannian 3-manifold of constant curvature.)

Harmonic maps from surfaces with indefinite metric, though of interest to physicists (see [27]), are far less understood then those from a surface with Riemannian metric. Meanwhile, the classical geometry of surfaces in $M^{3}$ has yet to be fully explored. The complex analytic techniques developed in this paper have applications in both areas.

In particular, we use holomorphic quadratic differentials to characterize harmonic maps from a surface $S$ with nondegenerate prescribed and induced metric, thereby associating to any such map three different metrics which are flat whenever they are nondegenerate. Replacing the Cauchy-Riemann equations by the Codazzi-Mainardi equations, we obtain in Theorems $\mathrm{A}$ and $\mathrm{B}$ different versions of the characterizations which can be applied using arbitrary coordinates on $S$. Theorem 7 explains the connection between harmonic maps and the sine-Gordon, sinh-Gordon and coshGordon equations, thereby clarifying the appearance of these equations as the theorem egregium formula relating intrinsic curvature and $K$ on surfaces in $E^{3}$ or $M^{3}$ with constant $K$ and indefinite second fundmental form II. (See [5] and Remark 7.)

On surfaces in $M^{3}$ with constant $H$ or $K \neq 0$, we provide special local coordinates and metrics which are flat wherever nondegenerate. This allows us to establish some statements in-the-large for surfaces of constant $H$ or $K \neq 0$ which are complete in one sense or another. We show that the Gauss map of a surface in $M^{3}$ is harmonic if and only if $H$ is constant, thus extending to $M^{3}$ the result [31] of Ruh and Vilms, that a hypersurface in $E^{n}$ has harmonic Gauss map if and only if $H$ is constant. By way of contrast, we show that where II is nondegenerate on a surface $S$ in $E^{3}$ or $M^{3}$, the Gauss map from $(S$, II) is harmonic if and only if $K$ is constant. Though Treibergs [34] has provided a wealth of examples of entire, spacelike surfaces in $M^{3}$ with constant $H$, we show that $K$ assumes values arbitrarily close to $H^{2}$ on such a surface, unless it is a hyperbolic cylinder.

The results in this paper indicate a bizarre similarity in the properties of surfaces of constant $H$ as compared with those of constant $K \neq 0$. This phenomenon is explained by the formalisms developed in $\$ 2-\$ 4$, which lie at the heart of all arguments. For other applications of these formalisms, see [26].

The claims made in $\$ 2$ can be easily checked by the reader. To clarify exposition, we defer proofs of most results in $\S 3-\S 6$ to $\S 7$. Wherever possible, $C^{\infty}$ smoothness is assumed. The symbol $c$ always denotes a real constant.

2. Preliminary notions. Throughout this paper, $S$ is an oriented surface without boundary. Suppose

$$
A=E d x^{2}+2 F d x d y+G d y^{2} \text { and } B=L d x^{2}+M d x d y+N d y^{2}
$$

are real quadratic forms on $S$. If $A$ is nondegenerate, call $A, B$ a pair, definite if $A$ is definite, and indefinite if $A$ is indefinite. The mean, extrinsic and skew curvatures $H=H(A, B), K=K(A, B)$ and $H^{\prime}=H^{\prime}(A, B)$ for a pair are defined by

$$
2 H=\operatorname{tr}_{A} B=\frac{E N+G L-2 M F}{\operatorname{det} A}, \quad K=\frac{\operatorname{det} B}{\operatorname{det} A}, \quad H^{\prime}=\left(H^{2}-K\right)^{1 / 2} .
$$


If $A$ and $B$ are both indefinite, $H^{\prime}$ can be imaginary, in which case we require that $i H^{\prime}<0$. Directions in which $A=0$ are called null directions, while those in which $B=0$ are called asymptotic directions. A point where $B \propto A$ is called an umbilic. Here, $H^{\prime}$ can vanish even at nonumbilics as the example $A=d x d y+d y^{2}, B=d x d y$ shows. If $H^{\prime}=0$ at a nonumbilic, then $A$ and $B$ must share exactly one null direction. Thus if $A$ or $B$ is definite, $H^{\prime}=0$ only at umbilics.

Whenever $H^{\prime}>0$ there are, locally, doubly orthogonal coordinates $x, y$ in terms of which

$$
A=E d x^{2}+G d y^{2}, \quad B=k_{1} d x^{2}+k_{2} d y^{2},
$$

where the principal curvatures $k_{1}$ and $k_{2}$ are real-valued functions given in one order or the other by $H \pm H^{\prime}$, so that

$$
2 H=k_{1}+k_{2}, \quad K=k_{1} k_{2}, \quad 2 H^{\prime}= \pm\left(k_{2}-k_{1}\right) .
$$

Where $H^{\prime}$ is imaginary, $H^{2}<K$, and the asymptotic directions separate the null directions. Moreover, there is no simultaneous real diagonalization of $A$ and $B$ as in (1). Instead, there is the simultaneous complex diagonalization given below in (14). Of course, anywhere on the interior of the umbilic set, coordinates which diagonalize $A$ do the same for $B$.

Various quadratic forms can be associated with a pair $A, B$. The skew forms $A^{\prime}=A^{\prime}(A, B)$ and $B^{\prime}=B^{\prime}(A, B)$ are defined only where $H^{\prime} \neq 0$ by

$$
H^{\prime} A^{\prime}=B-H A, \quad H^{\prime} B^{\prime}=H B-K A .
$$

The equivalent $C=C(A, B)$ of a third fundamental form and its skew analog $C^{\prime}=C^{\prime}(A, B)$ are given by

$$
C=2 H B-K A, \quad C^{\prime}=2 H B^{\prime}-K A^{\prime} .
$$

Wherever $K \neq 0$, det $C=K^{2} \operatorname{det} A$ and $\operatorname{det} C^{\prime}=K^{2} \operatorname{det} A^{\prime}$. The form $W$ is defined anywhere on $S$ by

$$
|\operatorname{det} A|^{1 / 2} W=\left|\begin{array}{ccc}
d y^{2} & -d x d y & d x^{2} \\
E & F & G \\
L & M & N
\end{array}\right|,
$$

so $\operatorname{det} W=\left(K-H^{2}\right)|\operatorname{det} A|$. Where $H^{2}<K, W$ is definite. Where $H^{2}>K$, the equation $W=0$ is solved by the pair of directions orthogonal for both $A$ and $B$. Where $H^{2}=K$, either $W \equiv 0$ because $B \propto A$, or else $W=0$ for the single null direction shared by $A$ and $B$.

If $A$ and $B$ are both nondegenerate then $B, A$ is a pair with

$$
\begin{gathered}
H(B, A)=H / K, \quad K(B, A)=1 / K, \quad H^{\prime}(B, A)=H^{\prime} /|K|, \\
A^{\prime}(B, A)= \pm A^{\prime}, \quad B^{\prime}(B, A)= \pm B^{\prime},
\end{gathered}
$$

where \pm is the sign of $K$. If $C$ is nondegenerate, then $C, B$ is a pair with the same curvature functions as $B, A$ and $C(C, B)=A$. When $H^{2}<K$ the forms $W=$ $W(A, B), W(B, C)$ and $W(C, B)$ all determine the same Riemann surface $R_{W}$ on $S$, as do $W(A, C)$ and $W(C, A)$ also if $H \neq 0$. 
The intrinsic curvature $K(A)$ is computed by viewing $A$ as a pseudo-Riemannian metric on $S$. (Use (3-6) in [33].) When $F=0$,

$$
4(E G)^{2} K(A)=G_{x}(E G)_{x}+E_{y}(E G)_{y}-2 E G\left(G_{x x}+E_{y y}\right) .
$$

When $E$ and $G$ are constants,

$$
\left(E G-F^{2}\right)^{2} K(A)=\left(E G-F^{2}\right) F_{x y}+F F_{x} F_{y} .
$$

We call $A, B$ a Codazzi pair, and write $\operatorname{Cod}(A, B)$ in case det $A \neq 0$ and

$$
\begin{aligned}
& L_{y}-M_{x}=L \Gamma_{12}^{1}+M\left(\Gamma_{12}^{2}-\Gamma_{11}^{1}\right)-N \Gamma_{11}^{2}, \\
& M_{y}-N_{x}=L \Gamma_{22}^{1}+M\left(\Gamma_{22}^{2}-\Gamma_{12}^{1}\right)-N \Gamma_{12}^{2},
\end{aligned}
$$

where the Christoffel symbols are computed for $A$. When using doubly orthogonal coordinates, $(9)$ reduces to

$$
L_{y}=E_{y} H, \quad N_{x}=G_{x} H
$$

or, equivalently, to

$$
\pm\left(k_{1}\right)_{y} E=E_{y} H^{\prime}, \quad \mp\left(k_{2}\right)_{x} G=G_{x} H^{\prime}
$$

where \pm is the sign of $k_{2}-k_{1}$.

When $H^{2}<K$, using coordinates $x, y$ which are isothermal for $W$, one has

$$
\begin{gathered}
A=E\left(d x^{2}-d y^{2}\right)+2 F d x d y, \quad B=L\left(d x^{2}-d y^{2}\right)+2 M d x d y, \\
\left(E^{2}+F^{2}\right)^{1 / 2} W=(E M-F L)\left(d x^{2}+d y^{2}\right)
\end{gathered}
$$

with $E M \neq F L$. Equations (9) thus become

$$
L_{x}+M_{y}=\left(E_{x}+F_{y}\right) H, \quad L_{y}-M_{x}=\left(E_{y}-F_{x}\right) H .
$$

In terms of the conformal parameter $z=x+i y$ on the Riemann surface $R_{W}$ determined by $W$ on $S$, (12) gives

$$
A=\varepsilon d z^{2}+\bar{\varepsilon} d \bar{z}^{2}, \quad B=\varrho d z^{2}+\bar{\complement} d \bar{z}^{2}=k \mathscr{E} d z^{2}+\overline{k \varepsilon} d \bar{z}^{2}
$$

where $2 \mathcal{E}=E-i F$ and $2 \mathcal{L}=2 k \mathcal{E}=L-i M$. Thus, setting $k_{1}=k$ and $k_{2}=\vec{k},(2)$ remains valid when $H^{2}<K$. The intrinsic curvature $K(A)$ is given here by

$$
4|\mathcal{E}|^{4} K(A)=\overline{\mathcal{E}}_{z}|\mathcal{E}|_{\bar{z}}^{2}+\mathcal{E}_{\bar{z}}|\overline{\mathcal{E}}|_{z}^{2}-2|\mathcal{E}|^{2}\left(\overline{\mathcal{E}}_{z z}+\mathcal{E}_{\bar{z} \bar{z}}\right)
$$

where $2 \partial / \partial z=\partial / \partial x-i \partial / \partial y$ and $2 \partial / \partial \bar{z}=\partial / \partial x+i \partial / \partial y$. Moreover, either of the equations

$$
\varrho_{\bar{z}}=\varepsilon_{\bar{z}} H, \quad \overline{\mathcal{E}}_{z}=\bar{\varepsilon}_{z} H
$$

expresses the condition $\operatorname{Cod}(A, B)$. The same is true for the reformulation

$$
\pm k_{\bar{z}} \mathcal{E}=\varepsilon_{\bar{z}} H^{\prime}, \quad \mp \bar{k}_{z} \overline{\mathcal{E}}=\bar{\varepsilon}_{z} H^{\prime},
$$

where \pm is the sign of $i(k-\bar{k})$. Note the formal similarity of (14)-(17) to (2), (7), (10) and (11).

The skew forms are best understood as follows. If $H^{2}>K$, doubly orthogonal coordinates give (1) and

$$
\pm A^{\prime}=-E d x^{2}+G d y^{2}, \quad \pm B^{\prime}=-k_{1} E d x^{2}+k_{2} G d y^{2}
$$


where \pm is the sign of $k_{1}-k_{2}$. If $H^{2}<K$, coordinates isothermal for $W$ give (12) and

$$
\pm i A^{\prime}=F\left(d y^{2}-d x^{2}\right)+2 E d x d y, \quad \pm i B^{\prime}=M\left(d y^{2}-d x^{2}\right)+2 L d x d y
$$

where \pm is the sign of $E M-F L$, which determines whether $W$ is positive or negative definite. If $z=x+i y$ is a conformal parameter on $R_{W},(14)$ and (19) give

$$
\pm A^{\prime}=-\mathcal{E} d z^{2}+\bar{\varepsilon} d \bar{z}^{2}, \quad \pm B^{\prime}=-k \mathcal{E} d z^{2}+\bar{k} \bar{\varepsilon} d \bar{z}^{2}
$$

with \pm as in (19). Where $H^{\prime} \neq 0$, we can think of $A^{\prime}, B^{\prime}$ as a pair, even though both forms are imaginary when $H^{\prime}$ is. Using (18)-(20), it is easy to check that

$$
\begin{aligned}
& H\left(A^{\prime}, B^{\prime}\right)= H(A, B), \quad K\left(A^{\prime}, B^{\prime}\right)=K(A, B), \\
& H^{\prime}\left(A^{\prime}, B^{\prime}\right)=H^{\prime}(A, B), \\
& \operatorname{det} A / \operatorname{det} A^{\prime}=\operatorname{det} B / \operatorname{det} B^{\prime}=\operatorname{det} C / \operatorname{det} C^{\prime}=-1 .
\end{aligned}
$$

When $H^{\prime}>0$, this makes $A$ (resp. $B$ or $C$ ) definite if and only if $A^{\prime}$ (resp. $B^{\prime}$ or $C^{\prime}$ ) is indefinite, and visa versa. However, when $H^{\prime}$ is imaginary, all the real quadratic forms $A, B, C, i A^{\prime}, i B^{\prime}$ and $i C^{\prime}$ are indefinite. Note that $C^{\prime}=C\left(A^{\prime}, B^{\prime}\right)$.

We denote by $R_{A}$ the Riemann surface determined on $S$ by a definite form $A$. The symbol $R$ is used to denote an arbitrary Riemann surface defined on $S$. Given some fixed $R$, and a real quadratic form $B=L d x^{2}+2 M d x d y+N d y^{2}$ on $S$, consider the quadratic differential defined by

$$
\Omega=\Omega(B, R)=(L-N-2 i M) d z^{2}
$$

using coordinates $x, y$ on $S$ so that $z=x+i y$ is a conformal parameter on $R$. Note that $\Omega\left(A, R_{A}\right) \equiv 0$. One calls $\Omega$ holomorphic if the coefficient $L-N-2 i M$ is complex analytic for every choice of $z$ on $R$.

While there are generally lots of holomorphic quadratic differentials available on any $R$ (see [32]), especially interesting situations arise when the $\Omega$ associated with some form of geometric significance on an immersed surface is holomorphic with respect to some conformal structure related to the immersion. The first such example was given by $\mathrm{H}$. Hopf in [12], and while numerous further examples are detailed in this paper, many other examples can be found in the literature.

Our first result states that conformal variation of a pair $A, B$ leads to a conformal change in $A^{\prime}, B^{\prime}, C$ and $W$.

LEMmA 1. If $\tilde{A}=\lambda A$ and $\tilde{B}=\mu B$ for a pair $A, B$ and functions $\lambda \neq 0$ and $\mu \neq 0$, then

$$
\lambda \tilde{H}=\mu H, \quad \lambda^{2} \tilde{K}=\mu^{2} K, \quad|\lambda| \tilde{H}^{\prime}=|\mu| H^{\prime}
$$

so that $\tilde{H}^{\prime} H^{\prime} \geqslant 0$, while

$$
\tilde{A}^{\prime}= \pm \lambda A^{\prime}, \quad \tilde{B^{\prime}}= \pm \mu B^{\prime}, \quad \lambda \tilde{C}=\mu^{2} C, \quad \tilde{W}= \pm|\mu| W,
$$

where \pm is the sign of $\mu \lambda$.

3. Codazzi pairs. In this section we extend to all Codazzi pairs some observations established in [19 and 20] for definite Codazzi pairs. The fundamental forms I, II of a spacelike or timelike surface in a pseudo-Riemannian 3-manifold of constant curvature are the most famous example of a Codazzi pair. (See [28] or [30], and [8].) 
Other examples are provided in results below. While it is standard to refer to $B$ as a Codazzi tensor on $S$ provided with the metric $A$, we talk about the Codazzi pair $A, B$ in order to emphasize that neither $A$ nor $B$ is fixed. Beyond that, we wish to hint at a rough symmetry in the roles of $A$ and $B$ illustrated by the material in $\S 4$.

Fact 1. If $\operatorname{Cod}(A, B)$ with $B \propto A$, then $B \equiv c A$.

Fact 2. Wherever $H^{\prime} \neq 0, \operatorname{Cos}(A, B)$ if and only if $\operatorname{Cod}\left(A^{\prime}, B^{\prime}\right)$.

Fact 3. Wherever $K \neq 0, \operatorname{Cod}(A, B)$ if and only if $\operatorname{Cod}(C, B)$.

For any nondegenerate $A, \operatorname{Cod}(A, A)$ is automatic. Given constants $\alpha$ and $\beta$, $\operatorname{Cod}(\alpha A, \beta B)$ follows from $\operatorname{Cod}(A, B)$ if $\alpha \neq 0$, while $\operatorname{Cod}(A, \alpha X+\beta Y)$ follows from $\operatorname{Cod}(A, X)$ and $\operatorname{Cod}(A, Y)$. However, conformal variation of a Codazzi pair does not generally lead to another Codazzi pair.

Fact 4. If $\operatorname{Cod}(A, B)$ and $\operatorname{Cod}(\lambda A, B)$ for a function $\lambda \neq 0$, then $\lambda$ is constant if $H \neq 0$.

Fact 5. If $\operatorname{Cod}(A, B)$ and $\operatorname{Cod}(A, \mu B)$ for a function $\mu \neq 0$, then $\mu$ is constant if $K \neq 0$.

Fact 6. If $\operatorname{Cod}(A, B)$ and $\operatorname{Cod}(B, A)$ then $K(A, B) \neq 0$ while $K(A) \equiv K(B) \equiv 0$ on the closure of the set where $H^{\prime}(A, B) \neq 0$.

The following formula was first obtained by Wegner in the Riemannian case. (See [35 or 17].)

Fact 7. If $\operatorname{Cod}(A, B)$, then $K(C)=K(A) / K$ wherever $K \neq 0$.

Taking $A=\mathrm{I}, B=\mathrm{II}$ and $C=\mathrm{III}$ for a surface $S$ in $M^{3}$, Fact 7 gives the theorem egregium equation $K(\mathrm{I})=\mp K(\mathrm{I}, \mathrm{II})$ where \pm is the sign of det $\mathrm{I}$. This follows since the Gauss map is to a sphere with indefinite (resp. definite) metric III of intrinsic curvature 1 (resp. - 1 ) if $I$ is indefinite (resp. definite). This is explained more fully in $\S 6$.

REMARK 1. Suppose that I, II and III are the fundamental forms for an immersion of $S$ in a pseudo-Riemannian 3-manifold of constant curvature, with I definite (resp. indefinite) and $K(\mathrm{I}) \equiv-1$ (resp. 1). Then there exists locally a usually different immersion of $S$ in $M^{3}$ with III as its first fundamental form and II as its second. This follows since Fact 3 gives Cod(III, II) while Fact 7 gives $K($ III $)=-1 / K(\mathrm{I}, \mathrm{II})$ (resp. $1 / K(\mathrm{I}, \mathrm{II})$ ), so that $K(\mathrm{III})=-K(\mathrm{III}, \mathrm{II})$ (resp. $K(\mathrm{III}, \mathrm{II})$ ) by (6). The fundamental theorem for surfaces (see $\S 117$ in [30]) thus guarantees the immersion in $M^{3}$ as described.

Remark 1 should be compared with Remark 1 in [20]. These two observations give a method for generating examples of surfaces with constant $K$ in $E^{3}$ and $M^{3}$.

The next statement is suggested by comparing Lemmas 9 and 10 in $\S 4$.

Fact 8. If $H K \neq 0$, then $\operatorname{Cod}(H A, B)$ is equivalent to $\operatorname{Cod}\left(B,|K|^{1 / 2} A\right)$.

4. Formal results. The material in this section was established in [20] for definite pairs. The results here pinpoint the role of the Codazzi-Mainardi equations in 
producing holomorphic quadratic differentials on surfaces with constant $H$ or $K$. If $\operatorname{Cod}(A, B)$ is given, the condition $H \equiv c$ plays the role for the pair $A, B$ which the condition $K \equiv c \neq 0$ plays for the pair $B, A$.

Lemma 2 is a reformulation of an observation $H$. Hopf made to show that all genus zero soap bubbles in $E^{3}$ are spheres. (See [12].)

Lemma 2. If $A, B$ is a definite pair, then any two conditions below imply the remaining two, except that one needs $H \neq 0$ with (ii) and (iv) to get (i) and (iii), and $H^{\prime} \neq 0$ with (iii) and (iv) to get (i) and (ii).

(i) $\operatorname{Cod}(A, B)$,

(ii) $H \equiv c$,

(iii) $\Omega\left(B, R_{A}\right)$ holomorphic,

(iv) $\Omega\left(C, R_{A}\right)$ holomorphic.

Lemma 3. If $A, B$ is an indefinite pair with $H^{2}>K$, then any two conditions below imply the remaining two, except that one needs $H \neq 0$ with (iv) and (ii) to get (i) and (iii).

(i) $\operatorname{Cod}(A, B)$,

(ii) $H \equiv c$,

(iii) $\Omega\left(B^{\prime}, R_{A^{\prime}}\right)$ holomorphic,

(iv) $\Omega\left(C^{\prime}, R_{A^{\prime}}\right)$ holomorphic.

Lemma 4. If $A, B$ is an indefinite pair with $H^{2}<K$, then any two conditions below imply the third.

(i) $\operatorname{Cod}(A, B)$,

(ii) $H \equiv c$,

(iii) $\Omega\left(H^{\prime} A^{\prime}, R_{W}\right)$ holomorphic.

Drawing Lemmas 2 through 4 together, we have the following result.

Theorem 1. If $\operatorname{Cod}(A, B)$ and $H \equiv c$, then $H^{\prime} A, H^{\prime} A^{\prime}$ and $W$ are flat wherever $H^{\prime} \neq 0$. In fact, there are local coordinates where $H^{\prime} \neq 0$ in terms of which $H^{\prime} A, H^{\prime} A^{\prime}$ and $W$ all have constant coefficients.

Just as $A$ was definite in Lemma 2, and indefinite in Lemmas 3 and 4, note that below, $B$ is definite in Lemma 5 and indefinite in Lemmas 6 and 7. Lemma 5 is a reformulation of an observation made in [13] to prove Liebmann's theorem that any compact $S$ in $E^{3}$ with $K \equiv c>0$ is a sphere.

Lemma 5. If $A, B$ is a pair, any two conditions below imply the remaining two, except that one needs $H^{\prime} \neq 0$ with (iii) and (iv) to get (i) and (ii).

(i) $\operatorname{Cod}(A, B)$,

(ii) $K \equiv c$, with $B$ definite,

(iii) $\Omega\left(A, R_{B}\right)$ holomorphic,

(iv) $\Omega\left(C, R_{B}\right)$ holomorphic.

Lemma 6. If $A, B$ is a pair with $H^{2}>K$, any two conditions below imply the remaining two.

(i) $\operatorname{Cod}(A, B)$, 
(ii) $K \equiv c$, with $B$ indefinite,

(iii) $\Omega\left(A^{\prime}, R_{B^{\prime}}\right)$ holomorphic,

(iv) $\Omega\left(C^{\prime}, R_{B^{\prime}}\right)$ holomorphic.

LEMMA 7. If $A, B$ is a pair with $H^{2}<K$, any two conditions below imply the third.

(i) $\operatorname{Cod}(A, B)$,

(ii) $K \equiv c$,

(iii) $\Omega\left(H^{\prime} B^{\prime}, R_{W}\right)$ holomorphic.

Drawing Lemmas 5 through 7 together is this exact counterpart to Theorem 1.

THEOREM 2. If $\operatorname{Cod}(A, B)$ and $K \equiv c \neq 0$, then $H^{\prime} B, H^{\prime} B^{\prime}$ and $W$ are flat wherever $H^{\prime} \neq 0$. In fact, there are local coordinates where $H^{\prime} \neq 0$ in terms of which $H^{\prime} B, H^{\prime} B^{\prime}$ and $W$ all have constant coefficients.

The next lemma is a reformulation of an observation made by Hilbert to show that there is no $S$ immersed in $E^{3}$ with I complete and $K \equiv-1$. (See Appendix 1 in [16].) Coordinates are asymptotic for a pair $A, B$ if $L \equiv N \equiv 0$, and Tchebychev if $|E| \equiv|G| \equiv 1$. Note that (8) gives $K(A)$ for Tchebychev coordinates.

Lemma 8. For a pair $A, B$ any two conditions below imply the third.

(i) $\operatorname{Cod}(A, B)$,

(ii) $K \equiv c$ with $B$ indefinite and $H^{\prime} \neq 0$,

(iii) asymptotic Tchebychev coordinates are locally available.

REMARK 2. Suppose that $K(A) \equiv c$ and that $x, y$ are Tchebychev coordinates. Setting $u=x-y$ and $v=x+y$, there are three possibilities. If $A$ is definite, $E=G= \pm 1$ and $|F|<1$. The angle $\omega$ between $x$ and $y$ coordinate curves is given by $\cos \omega=F$, and (8) yields a sine-Gordon equation

$$
c \sin \omega=\omega_{u u}-\omega_{v v} .
$$

If $A$ is indefinite with $E \equiv G \equiv \pm 1$, then $H^{2}>K$, the angle $\omega>0$ is given by $\cosh \omega=|F|>1$, and (8) yields a sinh-Gordon equation

$$
c \sinh \omega=\omega_{u u}-\omega_{v v},
$$

where \pm is the sign of $F$. If $A$ is indefinite with $E \equiv-G \equiv \pm 1$, then $H^{2}<K$, and we define the angle $\omega$ by $\sinh \omega=F$, since no normal definition of $\omega$ is available. (See $\S 46$ in [30].) Thus (8) yields

$$
c \cosh \omega=\omega_{u u}-\omega_{v v} .
$$

If $H=H(A, B) \neq 0$, then $H(H A, B)=1$. Using the pair $H A, B$ in Lemmas 2, 3 and 4 gives the following result, which is the basis for Theorem $A$ in $\$ 5$.

Lemma 9. If $H \neq 0$ then $\operatorname{Cod}(H A, B)$ is equivalent to $\Omega\left(B, R_{A}\right)$ holomorphic if $A$ is definite, to $\Omega\left(B^{\prime}, R_{A^{\prime}}\right)$ holomorphic if $A$ is indefinite with $H^{2}>K$, and to $\Omega\left(H^{\prime} A^{\prime}, R_{W}\right)$ holomorphic if $H^{2}<K$.

If $K=K(A, B) \neq 0$, then $K\left(B,|K|^{1 / 2} A\right)= \pm 1$, with \pm the sign of $K$. Using the pair $B,|K|^{1 / 2} A$ in Lemmas 5, 6 and 7 and applying (6) and Lemma 1 to reexpress all forms and curvatures in terms of those defined for the pair $A, B$, one gets the following result, which is the basis for Theorem B in $\$ 5$. 
LEMma 10. If $K \neq 0$ then $\operatorname{Cod}\left(B,|K|^{1 / 2} A\right)$ is equivalent to $\Omega\left(B, R_{A}\right)$ holomorphic if $A$ is definite, to $\Omega\left(B^{\prime}, R_{A^{\prime}}\right)$ holomorphic if $A$ is indefinite with $H^{2}>K$, and to $\Omega\left(H^{\prime} A^{\prime}, R_{W}\right)$ holomorphic if $H^{2}<K$.

5. Harmonic immersions. In this section we characterize harmonic maps $f$ : $(S, h) \rightarrow(\mathfrak{N}, \mathcal{G})$ with nondegenerate prescribed metric $h$ into an arbitrary $q$-dimensional manifold $\mathfrak{N}$ with pseudo-Riemannian metric $\mathcal{G}$, assuming that the metric $\mathrm{I}=f^{*} \mathcal{G}$ induced on $S$ is nondegenerate, so that $f$ must be an immersion, and $q \geqslant 2$.

Theorems $\mathrm{A}$ and $\mathrm{B}$ give formulations of the characterizations which can be applied using arbitrary coordinates on $S$. They also emphasize the importance of proper normalization of $h$ within the conformal class of metrics which $h$ determines. Thus one has generally available for a harmonic map $f$ not only the energy 1 and equiareal metrics $\Gamma$ and $\Pi$ used in Theorems A and B, but the flat metric $\left|H^{\prime}\right| h$ provided by Corollary 1 .

Our initial discussion is much like $\$ 3$ in [10]. We use the standard summation convention.

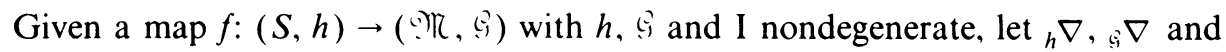
${ }_{1} \nabla$ be the associated Riemannian connections. (See [28 or 30].) Those who study harmonic maps refer to the covariant derivative $\nabla f_{*}$ given for vector fields $X$ and $Y$ on $S$ by

$$
\nabla f_{*}(X, Y)={ }_{\varsigma_{i}} \nabla_{f_{*}} f_{*} Y-f_{*}\left({ }_{h} \nabla_{X} Y\right)
$$

as the second fundamental form of $f$. (See $\$ 3.1$ in [7].) Here we follow the custom of geometers, taking as the second fundamental form of $f$ the normal vector field valued 2-form $B$ defined by the Gauss equation

$$
B(X, Y)={ }_{i:} \nabla_{f_{*}} f_{*} Y-f_{*}\left({ }_{I} \nabla_{X} Y\right) \text {. }
$$

Though $\nabla f_{*}$ is well defined even where $\mathrm{I}$ is degenerate, $B$ is not. Note that $B \equiv 0$ if $q=2$. Subtraction of (23) from (22) yields

$$
\nabla f_{*}(X, Y)=f_{*}\left({ }_{\mathrm{I}} \nabla_{X} Y-{ }_{h} \nabla_{X} Y\right)+B(X, Y),
$$

so that $B$ is just the normal component of $\nabla f_{*}$. Since further explicit reference to $\mathcal{G}$ is unnecessary, we write $f:(S, h) \rightarrow \mathfrak{T}$ henceforth.

In terms of local coordinates $x^{1}, x^{2}$ on $S$, and $y^{1}, y^{2}, \ldots, y^{q}$ on $\Re, h=h_{i j} d x^{i} d x^{j}$ and $\mathrm{I}=g_{i j} d x^{i} d x^{j}$ while the components of ${ }_{h} \nabla$ and ${ }_{\mathrm{I}} \nabla$ are given by the Christoffel symbols ${ }_{h} \Gamma_{j k}^{i}$ and $\Gamma_{\mathrm{I}}^{i}{ }_{j k}$. Thus (24) gives

$$
\left(\nabla f_{*}\right)_{i j}=B_{i j}+\left({ }_{\mathrm{I}} \Gamma_{i j}^{k}-{ }_{h} \Gamma_{i j}^{k}\right) f_{k}
$$

where $B_{i j}=B\left(\partial / \partial x^{i}, \partial / \partial x^{j}\right)$ and $f_{k}=\partial f / \partial x^{k}$.

The tension field $\tau$ of $f$ is defined (even if $\operatorname{det} \mathrm{I}=0$ ) by

$$
\tau=\operatorname{tr}_{h}\left(\nabla f_{*}\right)
$$

so that (25) gives

$$
\tau=h^{i j} B_{i j}+h^{i j}\left({ }_{\mathrm{I}} \Gamma_{i j}^{k}-{ }_{h} \Gamma_{i j}^{k}\right) f_{k}=\operatorname{tr}_{h} B+h^{i j}\left({ }_{\mathrm{I}} \Gamma_{i j}^{k}-{ }_{h} \Gamma_{i j}^{k}\right) f_{k} .
$$


We will call $\mathcal{F}^{h} \equiv \operatorname{tr}_{h} B$ the $h$-mean curvature vector field, since the ordinary mean curvature vector field is $\mathscr{H}=\mathcal{H}^{\mathrm{I}}$. By (27),

$$
\tau=\mathcal{H}^{h}+h^{i j}\left({ }_{\mathrm{I}} \Gamma_{i j}^{k}-{ }_{h} \Gamma_{i j}^{k}\right) f_{k},
$$

so that $\mathcal{H}^{h}$ is just the normal component of $\tau$. When $q=2, \mathcal{H}^{h} \equiv 0$.

The map $f:(S, h) \rightarrow \mathfrak{N}$ is harmonic if and only if $\tau \equiv 0$. The definition (26) of $\tau$ makes clear that the conformal class of $h$ rather than $h$ itself determines whether or not $f$ is harmonic. If det $h>0$, local $h$-isothermal coordinates can be used in terms of which $h_{11}=h_{22}$ and $h_{12}=0$, so that by (28), the condition $\tau \equiv 0$ reads

$$
0=\mathcal{H}^{h}+\left({ }_{\mathrm{I}} \Gamma_{11}^{k}+{ }_{\mathrm{I}} \Gamma_{22}^{k}\right) f_{k} \text {. }
$$

If det $h<0$, local $h$-null coordinates can be used in terms of which $h_{11}=h_{22}=0$, so that by (28), the condition $\tau \equiv 0$ reads

$$
0=\mathcal{H}^{h}+{ }_{\mathrm{I}} \Gamma_{12}^{k} f_{k} .
$$

Linear independence of the terms on the right sides of $(29)^{+}$and $(29)^{-}$gives the following.

LemmA $11^{+}$. If $\operatorname{det} h>0$ and $\operatorname{det} \mathrm{I} \neq 0$ for $f:(S, h) \rightarrow \mathfrak{M}$, then $f$ is harmonic if and only if

(i) $\mathcal{H}^{h} \equiv 0$, and

(ii) ${ }_{1} \Gamma_{11}^{1}+{ }_{\mathrm{I}} \Gamma_{22}^{1} \equiv_{\mathrm{I}} \Gamma_{11}^{2}+{ }_{\mathrm{I}} \Gamma_{22}^{2} \equiv 0$ for h-isothermal coordinates.

LEMma $11^{-}$. If $\operatorname{det} h<0$ and $\operatorname{det} \mathrm{I} \neq 0$ for $f:(S, h) \rightarrow \mathfrak{R}$, then $f$ is harmonic if and only if

(i) $\mathcal{K}^{h} \equiv 0$, and

(ii) ${ }_{\mathrm{I}} \Gamma_{12}^{1} \equiv{ }_{\mathrm{I}} \Gamma_{12}^{2} \equiv 0$ for $h$-null coordinates.

Theorems 3-5 provide characterizations of a harmonic $f:(S, h) \rightarrow$ ? $\mathbb{R}$ in terms of holomorphic quadratic differentials. We use the notation of $\S 2$, computing $H, K, H^{\prime}$, $h^{\prime}, \mathrm{I}^{\prime}$ and $W$ for the fixed pair $h, \mathrm{I}$. Theorem 3 generalizes the main result in [20], in which $\Re$ was assumed to be Riemannian. The fact that (ii) is a necessary condition there was first established in [6]. For the development of that observation, see the references in [7, §10.5] and [14].

TheOrem 3. A map $f:(S, h) \rightarrow \mathfrak{K}$ with definite $h$ and $\operatorname{det} \mathrm{I} \neq 0$ is harmonic if and only if

(i) $\mathcal{H}^{h} \equiv 0$, and

(ii) $\Omega\left(\mathrm{I}, R_{h}\right)$ is holomorphic.

Theorem 4. A map $f:(S, h) \rightarrow \mathfrak{M}$ with indefinite $h$, det $\mathrm{I} \neq 0$ and $H^{2}>K$ is harmonic if and only if

(i) $\mathcal{H}^{h} \equiv 0$, and

(ii) $\Omega\left(\mathrm{I}^{\prime}, R_{h^{\prime}}\right)$ is holomorphic.

Theorem 5. A map $f:(S, h) \rightarrow \mathfrak{N}$ with indefinite $h$, det $\mathrm{I} \neq 0$ and $H^{2}<K$ is harmonic if and only if

(i) $\mathcal{H}^{h} \equiv 0$, and

(ii) $\Omega\left(H^{\prime} h^{\prime}, R_{W}\right)$ is holomorphic. 
The characterizations above have a practical disadvantage. To check condition (ii), one has to work with special coordinates on $S$. Lemmas 9 and 10 provide reformulations which avoid this problem.

TheOREM A. A map $f:(S, h) \rightarrow \mathfrak{N}$ with $H K \neq 0$ is harmonic if and only if

(i) $\mathcal{H}^{h} \equiv 0$, and

(i) $\operatorname{Cod}(\Gamma, \mathrm{I})$ where $\Gamma=H h$.

THEOREM B. $A$ map $f(S, h) \rightarrow \mathfrak{M}$ with $K \neq 0$ is harmonic if and only if

(i) $\mathcal{H}^{h} \equiv 0$, and

(ii) $\operatorname{Cod}(\mathrm{I}, \Pi)$ where $\Pi=|K|^{1 / 2} h$.

The energy 1 metric $\Gamma$ has been quite useful in studying harmonic maps $f$ with definite prescribed metric $h$. (See [21-24].) It remains to be seen whether the equiareal metric $\Pi$ will be of similar value.

If $H^{\prime}=0$ and det $h<0$, we have no characterization of a harmonic $f$. Where $H^{\prime}=0$, either I $\propto h$, or else $h$ and I share exactly one null direction. If $\mathrm{I} \propto h, f$ is conformal, and we have the following statement, which is well known in the Riemannian case.

THEOREM 6. A conformal map $f:(S, h) \rightarrow$ M with $K \neq 0$ is harmonic if and only if $\mathfrak{H}^{1} \equiv 0$.

Theorems 1-5 have the following consequence.

Corollary 1. If $f:(S, h) \rightarrow$ ? I) is harmonic with $K H^{\prime} \neq 0$ then $H^{\prime} h . H^{\prime} h^{\prime}$ and $W$ are all flat. In fact, there are local coordinates in terms of which $H^{\prime} h, H^{\prime} h^{\prime}$ and $W$ all have constant coefficients.

This corollary shows that when $f$ is harmonic with $K H^{\prime} \neq 0$, then $R_{h}$ (resp. $R_{h^{\prime}}$ or $R_{W}$ ) is parabolic if $H^{\prime} h$ (resp. $H^{\prime} h^{\prime}$ or $W$ ) is a complete Riemannian metric. This leads to the following sort of result.

Corollary 2. Suppose $f:(S, h) \rightarrow$ OR is harmonic with det $h<0$ and I a complete Riemannian metric. Then $H^{\prime}$ is constant and $K\left(h^{\prime}\right) \equiv 0$ if either $K\left(h^{\prime}\right) \geqslant 0$ with $H^{\prime}$ bounded, or $K\left(h^{\prime}\right) \leqslant 0$ with $H^{\prime}$ bounded away from zero. Moreover, $K\left(\Pi^{\prime}\right) \equiv 0$ if $K\left(\Pi^{\prime}\right) \leqslant 0$.

The next result was suggested by an example from [29] as described in [9].

THEOREM 7. If $f:(S, h) \rightarrow \mathfrak{T}$ is harmonic with $\operatorname{det} h<0, H^{\prime} \neq 0$ and $K(\mathrm{I}) \equiv c$, there are local h-null coordinates $x, y$ on $S$ in terms of which $\mathbf{I}=g_{11} d x^{2}+2 g_{12} d x d y$ $+g_{22} d y^{2}$ with $\left|g_{11}\right| \equiv\left|g_{22}\right| \equiv 1$. If $u=x-y$ and $v=x+y$, the angle $\omega$ between $h$-null curves on $S$ satisfies the sine-Gordon equation

$$
c \sin \omega=\omega_{u u}-\omega_{v v}
$$

if $\operatorname{det} \mathrm{I}>0$, the sinh-Gordon equation

$$
\pm c \sinh \omega=\omega_{u u}-\omega_{v v}
$$


if $\operatorname{det} \mathrm{I}<0$ with $H^{2}>K$, and the cosh-Gordon equation

$$
c \cosh \omega=\omega_{u u}-\omega_{v v}
$$

if $\operatorname{det} \mathrm{I}<0$ with $H^{2}<K$.

Theorems 2-7 above, Corollary 1, and Theorem 10 in $\$ 6$ were announced in [25].

6. Surfaces in $M^{3}$. Minkowski 3-space $M^{3}$ is $R^{3}$ with the inner product $U \cdot V=$ $u_{1} v_{1}+u_{2} v_{2}-u_{3} v_{3}$ for vectors $U=\left(u_{1}, u_{2}, u_{3}\right)$ and $V=\left(v_{1}, v_{2}, v_{3}\right)$. This produces vectors of positive, imaginary and zero length, referred to as spacelike, timelike and null vectors, respectively. If the Euclidean angle a vector $V$ makes with the horizontal is less than $45^{\circ}, V$ is spacelike, if greater than $45^{\circ}, V$ is timelike, and if equal to $45^{\circ}, V$ is null. In any case, the square of Minkowski length $V \cdot V$ is never

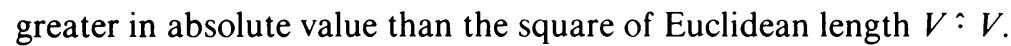

Given an immersion $X: S \rightarrow M^{3}$, surface theory can be done in the classical way so long as the induced metric $\mathrm{I}=d X \cdot d X$ is nondegenerate. We assume henceforth that $\varepsilon=\operatorname{det} \mathrm{I} \neq 0$, and write $\hat{\varepsilon}=\operatorname{det} \hat{I}$ for the identical immersion $\hat{X}: S \rightarrow E^{3}$. Note that $|\varepsilon| \leqslant|\hat{\varepsilon}|$. When $\varepsilon>0$, I is positive definite, all tangent vectors are spacelike, and $S$ is called a spacelike surface. If $\varepsilon<0$, two null directions divide the tangent plane into spacelike and timelike portions, and $S$ is called a timelike surface. Spacelike surfaces have timelike normal directions, and timelike surfaces have spacelike normal directions.

Writing $X_{j}=\partial X / \partial x^{j}$ for local coordinates $x^{1}, x^{2}$ on $S$, the vector product $X_{1} \times X_{2}$ has Minkowski length of absolute value $|\varepsilon|^{1 / 2}$, and Euclidean length $\hat{\varepsilon}^{1 / 2}$. The Minkowski unit normal $\nu$ is the reflection of $X_{1} \times X_{2}$ in the horizontal, divided by $|\varepsilon|^{1 / 2}$. Since the Euclidean unit normal $\hat{\nu}$ for $\hat{X}$ is $X_{1} \times X_{2}$ divided by $\hat{\varepsilon}^{1 / 2}$, the direction of $\nu$ is the reflection of the direction of $\hat{\nu}$ in the horizontal. The Gauss map $\nu: S \rightarrow \varsigma^{+}$of a spacelike surface is to the spacelike sphere $\varsigma^{+}$given by $u_{1}^{2}+u_{2}^{2}-u_{3}^{2}$ $=-1$. Note that $s^{+}$has intrinsic curvature -1 . The Gauss map $\nu: S \rightarrow \varsigma^{-}$of a timelike surface is to the timelike sphere $S^{-}$given by $u_{1}^{2}+u_{2}^{2}-u_{3}^{2}=-1$. Note that $\varsigma^{-}$has intrinsic curvature 1 . (See $\$ 87$ in [30].)

The second fundamental form II for $X$ is given by II $=-d X \cdot d \nu=b_{i j} d x^{i} d x^{j}$. There is little change in the Gauss equations

$$
X_{1,}=\Gamma_{1,}^{k} X_{k}-(\operatorname{sign} \varepsilon) h_{1,} \nu,
$$

where $\Gamma_{i j}^{k}$ is the standard Christoffel symbol associated with I, and $X_{i j}=\partial^{2} X / \partial x^{i} \partial x^{j}$. The Weingarten equations for $\nu_{i}=\partial \nu / \partial x^{i}$ are completely unaltered.

Throughout this section, we compute $H, K$, and $H^{\prime}$ for the pair I, II. Thus $H$ is the mean curvature, and $K$ the extrinsic curvature for the immersion $X: S \rightarrow M^{3}$. The forms III $=C(\mathrm{I}, \mathrm{II})=d \nu \cdot d \nu, \mathrm{I}^{\prime}, \mathrm{II}^{\prime}$ and $W$ are computed for the pair I, II as in $\S 2$. The corresponding curvatures and forms for $\hat{X}$ are indicated with a hatted symbol.

REMARK 3. Because $b_{i j}=X_{i j} \cdot \nu$, the relationship between $\nu$ and $\hat{\nu}$ described above gives

$$
|\varepsilon|^{1 / 2} \mathrm{II}=\hat{\varepsilon}^{1 / 2} \hat{\mathrm{II}}
$$


so that II $=\lambda$ Î with $\lambda \geqslant 1$ and $|K| \geqslant|\hat{K}|$. In particular, sign(det II) gives the same information as in the Euclidean case, with det II $>0$ implying strict local convexity. Thus II $\equiv 0$ holds only on a planar surface, and $K=0$ holds exactly where $\hat{K}=0$.

REMARK 4. A compact surface $S$ in $M^{3}$ cannot be entirely spacelike (or entirely timelike). Otherwise, the Euclidean normal directions would all make an angle of more than $45^{\circ}$ (or less than $45^{\circ}$ ) with the horizontal plane. A plane with its Euclidean normal in an omitted direction brought in toward $S$ would first touch $S$ so as to be tangent to it, producing a contradiction. Thus questions in-the-large about spacelike or timelike surfaces in $M^{3}$ involve open surfaces, and completeness of one sort or another is the natural global assumption. On a spacelike surface, I is Riemannian, and geodesic completeness has all the usual properties (see [11]), forcing Î to be complete as well. However, geodesic completeness of I on a timelike surface is not so definitive a notion. (See [1 and 37].) It may help to look for completeness of metrics which turn out to be definite on a timelike surface, such as $H^{\prime} \mathrm{II}^{\prime}$ if $H^{2}>K$ and $W$ if $H^{2}<K$. Moreover, completeness of $\hat{\mathrm{I}}$ is still a reasonable assumption.

The fundamental theorem for surfaces is little altered in $M^{3}$. (See [28 or 30].) In particular, Cod(I, II) and

$$
K(\mathrm{I})=-(\operatorname{sign} \varepsilon) K(\mathrm{I}, \mathrm{II})
$$

are necessary and sufficient conditions for the local existence of an $X: S \rightarrow M^{3}$ achieving given I, II as fundamental forms, with $X$ uniquely determined by I, II up to isometries of $M^{3}$.

We now state some results involving surfaces with constant $H$ in $M$. Extending $H$. Hopf's observation in [12], we have the following consequence of Lemmas 2-4.

LEMMA 12. On a surface in $M^{3}, H \equiv c$ is equivalent to $\Omega\left(\mathrm{II}, R_{\mathrm{I}}\right)$ holomorphic when $\varepsilon>0$, to $\Omega\left(\mathrm{II}^{\prime}, R_{\mathrm{I}^{\prime}}\right)$ holomorphic when $\varepsilon<0$ with $H^{2}>K$, and to $\Omega\left(H^{\prime} \mathrm{I}^{\prime}, R_{W}\right)$ holomorphic when $\varepsilon<0$ with $H^{2}<K$.

As in $E^{3}$, special coordinates are generally available, and certain metrics are flat. (See [15 and 36].)

LEMMA 13. If $H \equiv c$ on a surface in $M^{3}$, then wherever $\varepsilon H^{\prime}>0$, there are local coordinates $x, y$ such that

$$
H^{\prime} \mathrm{I}=d x^{2}+d y^{2}, \quad H^{\prime} \mathrm{II}=\left(H+H^{\prime}\right) d x^{2}+\left(H-H^{\prime}\right) d y^{2}
$$

wherever $\varepsilon H^{\prime}<0$, there are local coordinates $x, y$ such that

$$
H^{\prime} \mathrm{I}=d x^{2}-d y^{2}, \quad H^{\prime} \mathrm{II}=\left(H+H^{\prime}\right) d x^{2}-\left(H-H^{\prime}\right) d y^{2}
$$

and wherever $H^{\prime}$ is imaginary, there are local coordinates $x, y$ such that

$$
H^{\prime} \mathrm{I}= \pm i\left(d x^{2}-d y^{2}\right), \quad H^{\prime} \mathrm{II}=+i H\left(d x^{2}-d y^{2}\right)+2 H^{\prime} d x d y .
$$

Moreover, wherever $H^{\prime} \neq 0, H^{\prime} \mathrm{I}, H^{\prime} \mathrm{I}^{\prime}$ and $W$ are flat. 
REMARK 5. By the fundamental theorem for surfaces, the forms I, II given by (31), (32) or (33) with $H$ constant produce local imbeddings of the $x, y$-plane in $M^{3}$ with I, II as given if and only if the one function $H^{\prime}$ involved satisfies equation (30), since $\operatorname{Cod}(\mathrm{I}, \mathrm{II})$ is automatic. This gives a method for generating surfaces in $M^{3}$ with $H \equiv c$.

A surface in $M^{3}$ is called entire if it is the graph of a function over a whole plane. In [34], Treibergs constructs an abundance of entire, spacelike surfaces in $M^{3}$ with constant $H \neq 0$. On the other hand, Calabi shows in [3] that the plane is the only entire, spacelike surface in $M^{3}$ with $H \equiv 0$. The following result will be proved immediately, since the argument is typical of reasoning based on the methods in this paper. The cylinder mentioned is (up to isometries of $M^{3}$ ) the surface $u_{3}^{2}-u_{1}^{2}=$ $1 / 4 c^{2}$, with $u_{3}>0$.

THEOREM 8. An entire, spacelike $S$ in $M^{3}$ with $H \equiv c$ is a hyperbolic cylinder unless $K$ assumes values arbitrarily close to $H^{2}$ on $S$.

Proof. If $c=0$ then $K \equiv 0$, so assume $c \neq 0$. Then Cheng and Yau in [4] and Treibergs in [34] have shown that I is complete and $K \geqslant 0$ on any entire, spacelike $S$ in $M^{3}$ with $H \equiv c$. If $K$ is bounded away from $H^{2}$, then $H^{\prime}$ is bounded away from zero, and coordinates $x, y$ are locally available in terms of which (31) holds. Equation (30) thus reads $K(\mathrm{I})=H^{\prime} \Delta \log H^{\prime} / 2=-K \leqslant 0$, making $\log H^{\prime}$ superharmonic on $R_{\mathrm{I}}$. Since I is complete, the flat metric $H^{\prime} \mathrm{I}$ provided by Lemma 13 is also complete, which means that $R_{\mathrm{I}}$ is parabolic. Since $\log H^{\prime}$ is bounded from below, $H^{\prime}$ is constant, giving $K(\mathrm{I}) \equiv 0 \equiv K$ and $H= \pm H^{\prime}$. Thus $S$ has the fundamental forms of the spacelike hyperbolic cylinder, which proves the result.

REMARK 6. In the timelike case we have only weaker statements. If II is a complete Riemannian metric, with $\varepsilon<0$ and $H \equiv c$ on an $S$ in $M^{3}$, then $H^{\prime}$ is unbounded if $K\left(\mathrm{I}^{\prime}\right) \geqslant 0$ on $S$, and $1 / H^{\prime}$ is unbounded if $K\left(\mathrm{I}^{\prime}\right) \leqslant 0$ on $S$. If $H^{\prime} \mathrm{I}^{\prime}$ is a complete Riemannian metric on an $S$ in $M^{3}$ with $H \equiv c$, then $S$ is the timelike hyperbolic cylinder $u_{1}^{2}-u_{3}^{2}=1 / 4 c^{2}$ with $u_{1}>0$ (up to isometries of $M^{3}$ ) if either $K\left(\mathrm{I}^{\prime}\right) \geqslant 0$ with $H^{\prime}$ bounded on $S$, or $K\left(\mathrm{I}^{\prime}\right) \leqslant 0$ with $1 / H^{\prime}$ bounded on $S$.

The next two results associate harmonic maps with an $S$ in $M^{3}$ on which $H \equiv c$. The second statement suggests the possible extension to $M^{n}$ of a theorem of Ruh and Vilms (see [31]) which states that the Gauss map of a hypersurface of $E^{n}$ is harmonic if and only if $H \equiv c$.

THEOREM 9. The identity map $(S, \mathrm{I}) \rightarrow(S, \mathrm{II})$ of a surface $S$ in $E^{3}$ or $M^{3}$ is harmonic if and only if $H \equiv c$.

THEOREM 10. The Gauss map of a surface in $M^{3}$ is harmonic if and only if $H \equiv c$.

We now state some results involving surfaces with constant $K$ in $M^{3}$. See [13 and 20] for the analog in $E^{3}$ of the following consequence of Lemmas 5-7.

LEMma 14. On a surface in $M^{3}, K \equiv c \neq 0$ is equivalent to $\Omega\left(\mathrm{I}, R_{\mathrm{II}}\right)$ holomorphic when det II $>0$, to $\Omega\left(\mathrm{I}^{\prime}, R_{\mathrm{II}^{\prime}}\right)$ holomorphic when $\operatorname{det} \mathrm{II}<0$ with $H^{2}>K$, and to $\Omega\left(H^{\prime} \mathrm{II}^{\prime}, R_{W}\right)$ holomorphic when $\operatorname{det} \mathrm{II}<0$ with $H^{2}<K$. 
As in $E^{3}$, special coordinates are generally available, and certain metrics are flat. (See [13] and Appendix 1 in [16].)

LEMMA 15. If $K \equiv c \neq 0$ on a surface in $M^{3}$, then wherever $\operatorname{det} \mathrm{II} \cdot H^{\prime}>0$, there are local coordinates $x, y$ such that

$$
\pm H^{\prime} \mathrm{I}=\left(H+H^{\prime}\right) d x^{2}+\left(H-H^{\prime}\right) d y^{2}, \quad \pm H^{\prime} \mathrm{II}=K\left(d x^{2}+d y^{2}\right)
$$

wherever det II $<0$ and $\varepsilon>0$, there are local coordinates $x, y$ such that

$$
\mathrm{I}=d x^{2}+d y^{2}+2 \cos \omega d x d y, \quad \mathrm{II}=2|K|^{1 / 2} \sin \omega d x d y,
$$

with $0<\omega<\pi$; wherever det II $<0$ and $H^{\prime} \varepsilon<0$ there are local coordinates $x, y$ such that

$$
\mathrm{I}= \pm\left(d x^{2}+d y^{2}\right)+2 \cosh \omega d x d y, \quad \mathrm{II}= \pm 2|K|^{1 / 2} \sinh \omega d x d y
$$

with $0<\omega$ and independent sign choices; and wherever $H^{\prime}$ is imaginary, there are coordinates $x, y$ so that

$$
\mathrm{I}= \pm\left(d x^{2}-d y^{2}\right)+2 \sinh \omega d x d y, \quad \mathrm{II}=2|K|^{1 / 2} \cosh \omega d x d y .
$$

Moreover, wherever $H^{\prime} \neq 0, H^{\prime} \mathrm{II}, H^{\prime} \mathrm{II}^{\prime}$ and $W$ are flat.

Remark 7. Using Lemma 15 and Remark 2, the equation (30) for an $S$ in $M^{3}$ with $K \equiv c$ and det II $<0$ is a sine-Gordon equation if $\varepsilon>0$, a sinh-Gordon equation if $\varepsilon<0$ with $H^{2}>K$, and a cosh-Gordon equation if $\varepsilon<0$ with $H^{2}<K$. This phenomenon was essentially observed by Chern in [5].

REMARK 8. By the fundamental theorem for surfaces, the forms I, II given by (34)-(37) with $K \neq 0$ constant produce local imbeddings of the $x, y$-plane in $M^{3}$ with I, II as given if and only if the one function $H$ or $\omega$ involved satisfies (30), since $\operatorname{Cod}(\mathrm{I}, \mathrm{II})$ is automatic. This gives a method for generating surfaces in $M^{3}$ with $K \equiv c \neq 0$.

The following elementary observation will be proved immediately.

THEOREM 11. There is no complete, spacelike $S$ in $M^{3}$ with $K \leqslant c<0$.

Proof. Suppose there were such an $S$ in $M^{3}$. Since $\varepsilon>0$, (30) gives $K(\mathrm{I})>c>0$. But the theorem of Bonnet-Hopf-Rinow (see [11]) states that $S$ must be compact. By Remark 4, this is a contradiction.

REMARK 9. We have a weaker observation. If II (resp. II') is a Riemannian metric on an $S$ in $M^{3}$ with $K \equiv c<0$, then $K(\mathrm{II})>0$ (resp. $K\left(\mathrm{II}^{\prime}\right)>0$ ) somewhere on $S$, and $|H|$ is unbounded if $K(\mathrm{II}) \geqslant 0$ (resp. $K\left(\mathrm{II}^{\prime}\right) \geqslant 0$ ) on $S$.

The last two results associate harmonic maps with an $S$ in $M^{3}$ on which $K \equiv c \neq 0$.

THEOREM 12. The identity map $(S, \mathrm{II}) \rightarrow(S, \mathrm{I})$ for a surface in $E^{3}$ or $M^{3}$ with $\operatorname{det} \mathrm{II} \neq 0$ is harmonic iff $K \equiv c \neq 0$.

THEOREM 13. The Gauss map for a surface in $E^{3}$ or $M^{3}$ with det II $\neq 0$ is harmonic from $(S$, II) if and only if $K \equiv c \neq 0$. 
Because their proofs require only that Cod(I, II) hold, Lemmas 12-14, as well as Theorems 9 and 12, remain valid if $S$ is immersed with $\varepsilon \neq 0$ in a pseudo-Riemannian 3-manifold of constant curvature. (See [ 28 or 30].)

7. Proofs. Fact 1 is established under the same name in [20].

Proof of FACT 2. Use doubly orthogonal coordinates if $H^{2}>K$ and conformal parameters on $R_{W}$ if $H^{2}<K$ so that either (1), (18) and (11) hold, or else (14), (20) and (17). In either case, verification is immediate.

Proof of Fact 3. Use $H, K$ and $H^{\prime}$ for the pair $A, B$. We need only show that $\operatorname{Cod}(A, B)$ implies $\operatorname{Cod}(C, B)$ where $K \neq 0$. For $A$ is $C(C, B)$ wherever $K \neq 0$, while $K(C, B)=1 / K$. Thus our argument applies in the reverse direction as well. When $H^{\prime}=0$, there are two situations to consider. On any connected component of the interior of the umbilic set, $\operatorname{Cod}(A, B)$ gives $B=c A$ by Fact 1 , with $c \neq 0$ if $K \neq 0$. Thus $C=c^{2} A$, and $\operatorname{Cod}(C, B)$ follows there. On the interior of the set where $A$ and $B$ share exactly one null direction, use local coordinates in terms of which

$$
A=2 F d x d y, \quad B=2 M d x d y+N d y^{2}
$$

where $F \neq 0$ since $\operatorname{det} A \neq 0$. Then

$$
C=2\left(M^{2} d x d y+M N d y^{2}\right) / F
$$

with $M \neq 0$ where $K \neq 0$, so $\operatorname{Cod}(A, B)$ is expressed by

$$
M_{x} / M=F_{x} / F, \quad M_{y} / M-N_{x} / M=F_{y} / F,
$$

which easily gives $\operatorname{Cod}(C, B)$ where $K \neq 0$. A continuity argument completes the proof.

Proof of FaCt 4. If $H^{2}>K, \operatorname{Cod}(\lambda A, B)$ is given by

$$
L_{y}=\left(\lambda_{y} E+\lambda E_{y}\right) H / \lambda, \quad N_{x}=\left(\lambda_{x} G+\lambda G_{x}\right) H / \lambda
$$

in terms of doubly orthogonal coordinates. Subtraction of these equations from their counterparts in (10) yields $\lambda_{y} E H \equiv \lambda_{x} G H \equiv 0$, so that $H \operatorname{det} A \neq 0$ gives $\lambda$ constant. If $H^{2}<K$, use of conformal parameters on $R_{W}$ and the corresponding argument gives the same result.

Proof of FaCT 5. If $H^{2}>K, \operatorname{Cod}(A, \mu B)$ is given by

$$
\left(\mu L_{y}+\mu_{y} L\right) / \mu=E_{y} H, \quad\left(\mu N_{x}+\mu_{x} N\right) / \mu=G_{x} H
$$

in terms of doubly orthogonal coordinates. Subtraction of these equations from their counterparts in (10) yields $\mu_{x} N \equiv \mu_{y} L \equiv 0$, so that $\mu$ is constant if $K \neq 0$. If $H^{2}<K$, use of conformal parameters on $R_{W}$ and the corresponding argument gives the same result.

Proof of Faci 6. Use $H, K$ and $H^{\prime}$ for the pair $A, B$. Since $A$ and $B$ are nondegenerate, $K \neq 0$ is automatic. Where $H^{2}>K$, use of doubly orthogonal coordiantes, (10) and $\operatorname{Cod}(B, A)$ yields

$$
H L_{y}=E_{y} K, \quad H N_{x}=G_{x} K .
$$

On the interior of the $H \equiv 0$ set, and (10) and (41) give $L=L(x), N=N(y)$, $E=E(x)$ and $G=G(y)$. Where $H \neq 0$, multiplication of equations (41) by $H$ and subtraction of their counterparts in (10) gives $E_{y} H^{\prime 2} \equiv G_{x} H^{\prime 2} \equiv 0$, so that again, by 
(41) and $H^{\prime} \neq 0, L=L(x), N=N(y), E=E(x)$ and $G=G(y)$. Where $H^{2}<K$, repeat the argument using conformal parameters on $R_{W}$. By (7), $K(A) \equiv K(B) \equiv 0$ except on the boundary of the $H \equiv 0$ set. The result follows by continuity.

PROOF OF FACT 7 . Where $H^{\prime}>0$, use doubly orthogonal coordinates. Then $C=k_{1}^{2} E d x^{2}+k_{2}^{2} G d y^{2}$, while (11) gives

$$
\left(k_{2}^{2} G\right)_{x}=K G_{x}, \quad\left(k_{1}^{2} E\right)_{y}=K E_{y} .
$$

Substitution of this in the formula

$$
\begin{aligned}
4\left(K^{2} E G\right)^{2} K(C)= & \left(k_{2}^{2} G\right)_{x}\left(K^{2} E G\right)_{x}+\left(k_{1}^{2} E\right)_{y}\left(K^{2} E G\right)_{y} \\
& -2 K^{2} E G\left\{\left(k_{2}^{2} G\right)_{x x}+\left(k_{1}^{2} E\right)_{y y}\right\}
\end{aligned}
$$

for $K(C)$ yields $K(C)=K(A) / K$ if $K \neq 0$. by (7). Where $H^{\prime}$ is imaginary, use of conformal parameters on $R_{u}$ allows formal repetition of the argument. Where $H^{\prime}=0$, there are two situations to consider. On connected components of the interior of the umbilic set. Fact 1 gives $B=c A$ with $c \neq 0$ if $K \neq 0$. Thus $C=c^{2} A$ and $K(C)=K(A) / K$ if $K \neq 0$. On the interior of the set on which $A$ and $B$ share exactly one null direction, use local coordinates so that (38), (39) and (40) are valid. Then (8) gives

$$
F^{3} K(A)=F_{x} F_{y}-F F_{x y} .
$$

Equations (40) imply that $H$ and $K=H^{2}$ depend only on $y$ with

$$
M=F H=H N_{x} / H^{\prime},
$$

so that (39) gives $C=2 F H^{2} d x d y+2 H N d y^{2}$. Using (3-6) from [33] for $K(C)$ and (42) for $K(A)$, one verifies that $K(C)=K(\mathrm{I}) / K$ wherever $K \neq 0$, by (43).

ProOF OF FACT 8. Lemmas 9 and 10 give the result except where $A$ is indefinite with $H^{\prime}=0$. The reader can check the fact over any open connected set on which $B \propto A$ with $A$ indefinite, or on which $A$ and $B$ share exactly one null direction. A continuity argument then completes the proof.

Proof of Lemma 2. By Lemma 5 in [20], any two of the conditions (i), (ii) or (iii) imply the third and (iv) as well. Further,

$$
\Omega\left(C, R_{A}\right)=2 H \Omega\left(B, R_{A}\right) .
$$

Writing $\Omega\left(B, R_{A}\right)=\phi d z^{2}, \operatorname{Cod}(A, B)$ is given by

$$
2 \phi_{\bar{z}}=\lambda H
$$

on $R_{A}$, where $A=\lambda d z d \bar{z}$, with $\lambda \neq 0$ real. (See [12].) Suppose that (i) and (iv) hold. If $H \Omega\left(B, R_{A}\right) \equiv 0$, then $B \propto A$ on the $H \neq 0$ set. By Fact $1, \operatorname{Cod}(A, B)$ gives $H$ constant on each component of the $H \neq 0$ set. With $H \equiv 0$ elsewhere, continuity gives (ii) and thereby (iii) as well. Otherwise $H \Omega\left(B, R_{A}\right)=0$ only at isolated points. Elsewhere, local $z$ can be chosen on $R_{A}$ so that $H \phi \equiv 1$, making $\phi$ real. (See [2].) By (45) and $(H \phi)_{z} \equiv 0, H$ is constant except at isolated points. Again, continuity gives (ii) and thereby (iii) as well. Suppose next that (ii) and (iv) hold. If $H \neq 0$, (iii) is immediate, giving (i) as well. Finally, suppose that (iii) and (iv) hold. By (44), the real-valued function $H$ must be constant, unless $\Omega\left(B, R_{A}\right) \equiv 0$, so that $H^{\prime} \equiv 0$. If $H^{\prime} \neq 0$, (ii) holds, giving (i) as well. 
Proof of Lemma 3. Here $A$ is indefinite and $H^{2}>K$, so that $A^{\prime}$ is definite. Fact 2 shows that (i) is equivalent to $\operatorname{Cod}\left(A^{\prime}, B^{\prime}\right)$ while (21) shows that (ii) is equivalent to $H\left(A^{\prime}, B^{\prime}\right)$ constant, and that $H^{\prime}\left(A^{\prime}, B^{\prime}\right)=H^{\prime} \neq 0$ since $H^{2}>K$. Thus Lemma 2 applies to the definite pair $A^{\prime}, B^{\prime}$, and completes the proof.

Proof of Lemma 4. Use coordinates isothermal for $W$. Then (12) holds, and (13) can be rewritten to give

$$
\begin{aligned}
& (L-H E)_{y}+H_{y} E=(M-H F)_{x}+H_{x} F, \\
& (L-H E)_{x}+H_{x} E=-(M-H F)_{y}+H_{y} F,
\end{aligned}
$$

so that for any $z=x+i y$ on $R_{W}, \operatorname{Cod}(A, B)$ is expressed by

$$
[H F-M+i(H E-L)]_{\bar{z}}=(E+i F) H_{\bar{z}} \text {. }
$$

Since $H A-B=H^{\prime} A^{\prime}$ while $E^{2}+F^{2}=|\operatorname{det} A| \neq 0$, (46) implies that $H$ is constant if and only if $\Omega\left(H^{\prime} A^{\prime}, R_{W}\right)$ is holomorphic. Thus (i) and (ii) give (iii), while (i) and (iii) give (ii). But if (ii) and (iii) hold, both sides of (46) vanish, giving (i).

Proof of Theorem 1. Apply parts (i) and (ii) of the Main Lemma in [20] to the holomorphic quadratic differentials identified by Lemmas $2-4$.

Proof of Lemma 5. By Lemma 8 in [20], any two of the conditions (i), (ii) or (iii) imply the third, and (iv) as well. Given (i) and (iv), apply this observation to the pair $C, B$ which is Codazzi by Fact 3 , since $K(C, B)=1 / K \neq 0$. Thus $K(C, B)$ is constant, as is $K$, and (ii) follows because $B$ is definite, giving (iii) as well. If (ii) and (iv) hold, (iii) follows from

$$
\Omega\left(C, R_{B}\right)=-K \Omega\left(A, R_{B}\right)
$$

giving (i) as well. Finally, if (iii) and (iv) hold, note that since $B$ is definite, $K \neq 0$. By (47), $\Omega\left(C, R_{B}\right)=0$ only where $A \propto B$. Assuming that $H^{\prime} \neq 0$, (47) shows that $\Omega\left(C, R_{B}\right) \neq 0$ and $\Omega\left(A, R_{B}\right) \neq 0$ are both holomorphic if and only if the realvalued function $K$ is constant. Since $B$ is positive definite, (ii) holds, giving (i) as well.

Proof of Lemma 6. Any two of the conditions imply that $B$ is indefinite. Since $H^{2}>K,(21)$ shows that $B^{\prime}$ is definite with $K\left(A^{\prime}, B^{\prime}\right)=K$ and $H^{\prime}\left(A^{\prime}, B^{\prime}\right)=H^{\prime} \neq 0$. By Fact $2, \operatorname{Cod}(A, B)$ is equivalent to $\operatorname{Cod}\left(A^{\prime}, B^{\prime}\right)$. Thus Lemma 5 applied to the pair $A^{\prime}, B^{\prime}$ completes the proof.

Proof of Lemma 7. Since $H^{2}<K$, both $A$ and $B$ are indefinite and $K>0$. In asymptotic coordinates, $\operatorname{Cod}(A, B)$ is given by

$$
M_{x} / M=\Gamma_{11}^{1}-\Gamma_{12}^{2}, \quad M_{y} / M=\Gamma_{22}^{2}-\Gamma_{12}^{1},
$$

while $K$ is constant if and only if

$$
M_{x} / M=\Gamma_{11}^{1}+\Gamma_{12}^{2}, \quad M_{y} / M=\Gamma_{22}^{2}+\Gamma_{12}^{1},
$$

because $K=-M / \operatorname{det} A$, and always,

$$
\frac{(\operatorname{det} A)_{x}}{2 \operatorname{det} A}=\Gamma_{11}^{1}+\Gamma_{12}^{2}, \quad \frac{(\operatorname{det} A)_{y}}{2 \operatorname{det} A}=\Gamma_{22}^{2}+\Gamma_{12}^{1} .
$$

Given (i) and (ii), (48) and (49) hold, so that $\Gamma_{12}^{1}=\Gamma_{12}^{2}=0$, or

$$
E G_{x}=F E_{y}, \quad G E_{y}=F G_{x} .
$$


Multiplying equations (51) by $F$ and $E$, respectively, gives $E_{y} \operatorname{det} A \equiv 0$, or $E_{y} \equiv 0$. Multiplying by $G$ and $F$, respectively, gives $G_{x} \operatorname{det} A \equiv 0$, or $G_{x} \equiv 0$. Thus

$$
A=E(x) d x^{2}+2 F d x d y+G(y) d y^{2}
$$

with $0>H^{2}-K=M^{2} E G /(\operatorname{det} A)^{2}$, giving $E G<0$. Thus local asymptotic coordinates $x, y$ can be chosen so that

$$
A=d x^{2}+2 F d x d y-d y^{2}, \quad B=2 M d x d y .
$$

It is easy to check that $x, y$ are isothermal for $W$ and that

$$
\Omega\left(H^{\prime} B^{\prime}, R_{W}\right)=\Omega\left(H B-K A, R_{W}\right)=-2 K d z^{2}
$$

is holomorphic, since $K$ is constant. Therefore (i) and (ii) give (iii). Because $H^{\prime} B^{\prime}$ is indefinite in this lemma, $\Omega\left(H^{\prime} B^{\prime}, R_{W}\right) \neq 0$. Given just (iii), there are local parameters $z$ anywhere on $R_{W}$ in terms of which $\Omega\left(H^{\prime} B^{\prime}, R_{W}\right)=c d z^{2}$ for any prescribed real constant $c \neq 0$. Using such $z=x+i y, H^{\prime} \neq 0$ and (19) give $L=0$, so that $x, y$ are asymptotic coordinates isothermal for $W$. Thus (12) is valid with $L=0$, while (i) and (ii) are again expressed by (48) and (49). Given (iii) and (ii), take local $z$ on $R_{W}$ so that

$$
\Omega\left(H^{\prime} B^{\prime}, R_{W}\right)=\Omega\left(H B-K A, R_{W}\right)=-2 K E d z^{2}=-2 K d z^{2} .
$$

Thus $E=1, G=-E=-1, \Gamma_{12}^{2} \equiv \Gamma_{12}^{1} \equiv 0$, and (49) gives (48), so (ii) and (iii) give (i). If (i) and (iii) hold, take local $z$ on $R_{W}$ so that

$$
\Omega\left(H^{\prime} B^{\prime}, R_{W}\right)=-2 K E d z^{2}=-2 d z^{2} .
$$

Differentiating $K E=1$, using (50) and $K=-M^{2} \operatorname{det} A$, we get

$$
\begin{aligned}
& \frac{M_{x}}{M}=\frac{(\operatorname{det} A)_{x}}{2 \operatorname{det} A}-\frac{E_{x}}{2 E}=\Gamma_{11}^{1}+\Gamma_{12}^{2}-\frac{E_{x}}{2 E}, \\
& \frac{M_{y}}{M}=\frac{(\operatorname{det} A)_{y}}{2 \operatorname{det} A}-\frac{E_{y}}{2 E}=\Gamma_{22}^{2}+\Gamma_{12}^{1}-\frac{E_{y}}{2 E}
\end{aligned}
$$

so that (48) yields $2 \Gamma_{12}^{2}=E_{x} / 2 E$ and $2 \Gamma_{12}^{1}=E_{y} / 2 E$, or

$$
\left(F^{2}-E^{2}\right) E_{x}=2 E F E_{y}, \quad 2 E F E_{x}=\left(E^{2}-F^{2}\right) E_{y} .
$$

Since $K E=1 \neq 0$, we get $E_{x} \equiv E_{y} \equiv 0$ and $\Gamma_{12}^{2} \equiv \Gamma_{12}^{1} \equiv 0$. Thus (48) gives (49), so that (i) and (iii) give (ii).

Proof of Theorem 2. Use (6), and apply parts (i) and (ii) of the Main Lemma in [11] to the holomorphic quadratic differentials identified in Lemmas 5-7.

Proof of Lemma 8. Suppose (i) and (ii) hold. Then $B$ is indefinite, and the argument starting with the second sentence in the proof of Lemma 7 yields asymptotic coordinates in terms of which (52) is valid, with $E G \neq 0$ since, by (ii), $H^{\prime} \neq 0$. Thus asymptotic Tchebychev coordinates exist locally, so that (i) and (ii) give (iii). If (iii) holds, use the special coordinates indicated. Since they are asymptotic, (i) and (ii) are given by (48) and (49), respectively. However, since $E$ and $G$ are constant, $\Gamma_{12}^{1}=\Gamma_{12}^{2}=0$, making (48) and (49) equivalent. Thus, given (iii), either of the conditions (i) or (ii) implies the other.

In order to obtain Theorems 2-4, we need only reformulate condition (ii) in Lemma $11^{+}$or $11^{-}$, which states that the tangent component of the tension field $\tau$ vanishes. 
Proof of Theorem 3. Using the $h$-isothermal coordinates $x^{1}, x^{2}$ on $S$, the equations

$$
{ }_{\mathrm{I}} \Gamma_{11}^{1}+{ }_{\mathrm{I}} \Gamma_{22}^{1} \equiv{ }_{\mathrm{I}} \Gamma_{11}^{2}+{ }_{\mathrm{I}} \Gamma_{22}^{2} \equiv 0
$$

are the Cauchy-Riemann equations for the functions $g_{11}-g_{22}$ and $-2 g_{12}$, since

$$
2_{\mathrm{I}} \Gamma_{i j}^{k}=g^{k r}\left(\frac{\partial g_{r j}}{\partial x^{i}}+\frac{\partial g_{r i}}{\partial x^{j}}-\frac{\partial g_{i j}}{\partial x^{r}}\right) .
$$

Thus (ii) holds in Lemma $11^{+}$if and only if $\Omega\left(\mathrm{I}, R_{h}\right)$ is holomorphic.

To proceed further, we need the following interpretation of condition (ii) in Lemma $11^{-}$.

Lemma 16. Suppose a map $f:(S, h) \rightarrow \mathfrak{M}$ has indefinite $h$ and nondegenerate $\mathrm{I}$. Then there are local $h$-null coordinates for which ${ }_{1} \Gamma_{12}^{1} \equiv_{1} \Gamma_{12}^{2} \equiv 0$ if and only if there are local h-null coordinates $x, y$ such that where $H^{\prime} \neq 0$ is real,

$$
\mathrm{I}=\left(d x^{2}+d y^{2}\right)+2 F d x d y
$$

where $H^{\prime} \neq 0$ is imaginary,

$$
\mathrm{I}=d x^{2}-d y^{2}+2 F d x d y
$$

and where $H^{\prime}=0$,

$$
\mathrm{I}=\varepsilon d y^{2}+2 F d x d y
$$

with $|\varepsilon|=1$ or 0 .

Proof. Given condition (ii) in Lemma $11^{-}$, and $h$-null coordinates $x^{1}, x^{2},(53)$ gives

$$
g_{22} \frac{\partial g_{11}}{\partial x^{2}}=g_{12} \frac{\partial g_{22}}{\partial x^{1}}, \quad g_{11} \frac{\partial g_{22}}{\partial x^{1}}=g_{12} \frac{\partial g_{11}}{\partial x^{2}}
$$

Multiplying the first equation above by $g_{12}$ and the second by $g_{22}$ gives $\operatorname{det} \mathrm{I} \cdot \partial g_{22} / \partial x^{1} \equiv 0$. Multiplying the first equation above by $g_{11}$ and the second by $g_{12}$ gives det $\mathrm{I} \cdot \partial g_{11} / \partial x^{2} \equiv 0$. Since $\operatorname{det} \mathrm{I} \neq 0, g_{11}$ depends only on $x^{1}$ and $g_{22}$ depends only on $x^{2}$. Thus there are local $h$-null coordinates $u=u\left(x^{1}\right)$ and $v=v\left(x^{2}\right)$ such that $\mathrm{I}=E d u^{2}+2 F d u d v+G d v^{2}$ with $|E|=1$ or 0 and $|G|=1$ or 0 . But sign $E G=\operatorname{sign}\left(H^{2}-K\right)$, so that $E G=1$ if $H^{\prime} \neq 0$ is real, $E G=-1$ if $H^{\prime}$ is imaginary, and $E G=0$ if $H^{\prime}=0$. Thus either $u=x$ and $v=y$ or $u=y$ and $v=-x$ yield the coordinates described in the lemma. Argument in the reverse direction is brief, since ${ }_{\mathrm{I}} \Gamma_{12}^{1} \equiv_{\mathrm{I}} \Gamma_{12}^{2} \equiv 0$ for the $h$-null coordinates $x, y$.

It is easy to check that $h$-null coordinates which give (54) are $h^{\prime}$-isothermal, and that $h$-null coordinates which give (55) are $W$-isothermal. By Lemma 1 , this observation remains valid if $h$ is replaced by a conformally equivalent metric. The same can be said, of course, for conditions (i) and (ii) in Lemma $11^{+}$or $11^{-}$.

To establish Theorems 4 and 5, we will use the normalized metric $\Pi=|K|^{1 / 2} h$ which is conformally equivalent to $h$. Since det $I \neq 0, \Pi$ is nondegenerate. Moreover, $K(\mathrm{I}, \Pi) \equiv \pm 1$, where \pm is the sign of $K$ for the fixed pair $h$, I. This explains why $\Pi$ 
is called the equiareal metric for the map $f(S, h) \rightarrow \mathfrak{T}$. Note the use of $\Pi$ in Theorem B.

Proof of Theorems 4 And 5. Apply Lemma 8 to the pair I, $\Pi$. Where $H^{\prime} \neq 0$ (or, equivalently, where $H^{\prime}(I, \Pi) \neq 0$ ), there are local $h$-null coordinates giving (54) or (55) if and only if $\operatorname{Cod}(I, \Pi)$ holds. If $H^{2}>K$, use Lemmas 6 and 16 to show that (ii) in Lemma $11^{-}$is equivalent to $\left(\mathrm{I}^{\prime}, R_{\mathrm{II}^{\prime}}\right)$ holomorphic, where $\mathrm{I}^{\prime}$ and $\Pi^{\prime}$ are computed for the pair I, П. By Lemma 1 and formula (6), (ii) in Lemma $11^{-}$is equivalent to $\Omega\left(\mathrm{I}^{\prime}, R_{h^{\prime}}\right)$ holomorphic, computing $\mathrm{I}^{\prime}$ and $h^{\prime}$ for our fixed pair $h$, I. If $H^{2}<K$, use Lemmas 7 and 16 to show that (ii) in Lemma $11^{-}$is equivalent to $\Omega\left(H^{\prime} \Pi^{\prime}, R_{W}\right)$ holomorphic, computing $H^{\prime}, \Pi^{\prime}$ and $W$ for the pair I, $\Pi$. By Lemma 1 and formula (6), (ii) in Lemma $11^{-}$is equivalent to $\Omega\left(H^{\prime} h^{\prime}, R_{W}\right)$ holomorphic, computing $H^{\prime}, h^{\prime}$ and $W$ for our fixed pair $h$, I. Thus Theorems 3 and 4 follow from Lemma $11^{-}$.

Proof of Theorems A AND B. Lemmas 9 and 10 give the results except where $h$ is indefinite with $H^{\prime} \neq 0$. The reader can check the theorems over any open connected set on which $B \propto A$ with $A$ indefinite, or on which $A$ and $B$ share exactly one null direction. A continuity argument then completes each proof.

Proof of Theorem 6. Here, coordinates isothermal (resp. null) for $h$ are isothermal (resp. null) for I. Using (53), Lemmas $11^{+}$and $11^{-}$give the result.

Proof of Corollary 2. Since I is positive definite and det $h<0, h^{\prime}$ is positive definite. Thus we can apply Lemma 1 from [20] to the pair $h^{\prime}$, I using $H\left(h^{\prime}, \mathrm{I}\right)=H^{\prime}$ $=H^{\prime}(h, \mathrm{I})$ to show that $H^{\prime} h^{\prime}$ is complete because $\mathrm{I}$ is. By Corollary $1, H^{\prime} h^{\prime}$ is a complete flat metric, making $R_{h^{\prime}}$ parabolic. Taking coordinates for which $H^{\prime} h^{\prime}=$ $d x^{2}+d y^{2}$, we get

$$
2 K\left(h^{\prime}\right)=H^{\prime} \Delta \log H^{\prime} .
$$

Thus $\log H^{\prime}$ is subharmonic (resp. superharmonic) wherever $K\left(h^{\prime}\right) \geqslant 0$ (resp. $\leqslant 0$ ). The conclusions follow, with the last claim holding since $K(\Pi, I) \equiv-1$ forces $H^{\prime}(I, \mathrm{I})$ to be bounded away from zero.

Proof of TheOREM 7. Use the coordinates provided by Lemma 16 and Remark 2.

ProOfs of Lemmas 13 AND 15. As noted in [2], wherever a holomorphic quadratic differential $\Omega=\phi d z^{2} \neq 0$ on a Riemann surface $R$, there are local conformal parameters $z-x+i y$ in terms of which $\phi$ is any fixed complex constant $\neq 0$. The coordinates in these lemmas are obtained by taking $\phi \equiv 2$ or $\phi \equiv 2 i$.

Pro(ol: (ol: Ri:mARK 6. The first claim follows from Corollary 2 and Theorem 9. In the second claim. Theorem 9 and Corollary 1 make $H^{\prime} \mathrm{I}^{\prime}$ flat, so that $R_{\mathrm{I}^{\prime}}$ is parabolic, since $H^{\prime} \mathrm{I}^{\prime}$ is assumed to be complete. Taking $x, y$ so that $H^{\prime} \mathrm{I}^{\prime}=d x^{2}+d y^{2}$, we get $2 K\left(\mathrm{I}^{\prime}\right)=H^{\prime} \Delta \log H^{\prime}$, making $\log H^{\prime}$ subharmonic (resp. superharmonic) wherever $K\left(\mathrm{I}^{\prime}\right) \geqslant 0$ (resp. $\leqslant 0$ ). Thus $H^{\prime}$ is constant and $K\left(\mathrm{I}^{\prime}\right) \equiv 0$ if $K\left(\mathrm{I}^{\prime}\right) \geqslant 0$ with $H^{\prime}$ bounded, or $K\left(\mathrm{I}^{\prime}\right) \leqslant 0$ with $H^{\prime}$ bounded away from zero. By $(30), K(\mathrm{I}) \equiv K \equiv 0$ if $H^{\prime}$ is constant, so that I and II in (32) belong to the timelike hyperbolic cylinder.

Proof of Remark 9. The claim follows from Corollary 2, Theorem 12 and (6). For $H^{\prime}$ is automatically bounded away from zero, making $\mathrm{H}^{\prime} \mathrm{II}$ (resp. $\mathrm{H}^{\prime} \mathrm{II}^{\prime}$ ) a complete flat metric, and $R_{\mathrm{II}}$ (resp. $R_{\mathrm{II}}$ ) parabolic. Exactly as before, one gets $H^{\prime}$ constant if $K(\mathrm{II}) \leqslant 0$ (resp. $K\left(\mathrm{II}^{\prime}\right) \leqslant 0$ ), or if $K(\mathrm{II}) \geqslant 0$ (resp. $K\left(\mathrm{II}^{\prime}\right) \geqslant 0$ ) with $H$, 
and thereby $H^{\prime}$, bounded. But if $H^{\prime}$ is constant, so are $H$ or $\omega$, making $K(\mathrm{I}) \equiv K \equiv 0$, a contradiction.

Theorems 9 and 12 are easily proved by comparing Lemmas 12 and 14 (and their Euclidean equivalents) with Theorems 3, 4 and 5. It remains to check Theorem 10 and 13.

Proof of Theorem 10. There is a notational conflict here. If $H, K, H^{\prime}, \mathrm{I}^{\prime}, \mathrm{II}^{\prime}, \mathrm{III}$, III' and $W$ are to be computed for the fundamental pair I, II on $S$, then we need different names for the objects computed for the fixed pair I, III associated with the Gauss map $\nu$. Accordingly, we write $\tilde{H}=H(\mathrm{I}, \mathrm{III}), \tilde{K}=K(\mathrm{I}, \mathrm{III}), \tilde{H}^{\prime}=H^{\prime}(\mathrm{I}, \mathrm{III})$, $\tilde{\mathrm{I}}^{\prime}=\mathrm{I}^{\prime}(\mathrm{I}, \mathrm{III}), \mathrm{III}^{\prime}=\mathrm{III}^{\prime}(\mathrm{I}, \mathrm{III})$ and $\tilde{W}=W(\mathrm{I}, \mathrm{III})$, so that

$$
\tilde{H}^{\prime} \tilde{\mathrm{I}}^{\prime}=\mathrm{III}-\tilde{H} \mathrm{I}, \quad \tilde{H}^{\prime} \mathrm{I} \tilde{\mathrm{II}}{ }^{\prime}=\tilde{H} \mathrm{III}-\tilde{K} \mathrm{I},
$$

while

$$
H^{\prime} \mathrm{I}^{\prime}=\mathrm{II}-H \mathrm{I}, \quad H^{\prime} \mathrm{II}^{\prime}=H \mathrm{II}-K \mathrm{I} .
$$

Since III $=2 H \mathrm{II}-K \mathrm{I}$, it follows that

$$
\tilde{H}=2 H^{2}-K, \quad \tilde{K}=K^{2}, \quad \tilde{H}^{\prime}=2 H^{\prime}|H| .
$$

In particular, $\tilde{H}^{\prime}$ and $H^{\prime}$ are real or imaginary together, $\tilde{W} \propto W$, and

$$
\tilde{\mathrm{I}}^{\prime}= \pm \mathrm{I}^{\prime}, \quad \tilde{\mathrm{II}} \mathrm{I}^{\prime}= \pm \mathrm{III}^{\prime} .
$$

It is sufficient to prove the theorem on each open connected subset $U$ of $S$ on which one of the following holds:

(i) $\tilde{K} \neq 0, \tilde{H}^{2}>\tilde{K}$,

(ii) $\tilde{K} \neq 0, \tilde{H}^{2}<\tilde{K}$,

(iii) $\tilde{K} \neq 0$, III $\propto$ I,

(iv) $\tilde{K} \neq 0$, III $\not \subset \mathrm{I}, \tilde{H}^{2} \equiv \tilde{K}$,

(v) $\tilde{H} \equiv \tilde{K} \equiv 0$,

(vi) $\tilde{K} \equiv 0, \tilde{H} \neq 0$.

The union of such sets $U$ is an open dense subset of $S$. Since $S$ is assumed to be connected, the theorem follows by continuity. We devote a paragraph to each case listed above. When using Theorems 2-5, or Lemmas $11^{+}$and $11^{-}$, we ignore their condition (i), since the Gauss map $\nu$ is to a surface. Remember that Cod(I, II) holds.

In case (i), Lemma 2 and Theorem 3 give the result if $I$ is definite. If $I$ is indefinite, $\tilde{H}^{2}>\tilde{K}$ and (59) guarantee that $H^{\prime}>0$ and $H \neq 0$. Thus Lemma 3, Theorem 4 and (60) give the result.

In case (ii), I is indefinite with $H^{\prime} \neq 0$ imaginary and $H \neq 0$ by (59). Thus Lemma 4, Theorem 5 and (60) give $\nu$ harmonic if $H$ is constant. Conversely, if $\nu$ is harmonic, use the $\tilde{W}$ isothermal coordinates provided by Lemma 16 , so that III is given by (55) with $\mathrm{I}=2 m d x d y$. It follows that II $=L\left(d x^{2}-d y^{2}\right)+2 M d x d y$, with $H=M / m$, $K=\left(L^{2}+M^{2}\right) / m^{2}$ and $L=m / 2 M$. Thus $\operatorname{Cod}(\mathrm{I}, \mathrm{II})$ is given by

$$
M_{x}-(m / 2 M)_{y}=M m_{x}\left|m, \quad(m / 2 M)_{x}+M_{y}=M_{y}\right| m,
$$

which simplifies to

$$
2 M^{2}(M / m)_{x}+m(M / m)_{y}=0, \quad m(M / m)_{x}-2 M^{2}(M / m)_{y}=0 .
$$


Since $m \neq 0$, the only solution to this system is $(M / m)_{x} \equiv(M / m)_{y} \equiv 0$, so $H$ is constant.

In case (iii), $\nu$ is harmonic on $U$ by Theorem 6 . Thus we must show that $H$ is constant on $U$ as well. Where $H \neq 0$, III $\propto$ I gives II $\propto$ I, so that Fact 1 gives $H$ locally constant. Since $U$ is connected, continuity of $H$ gives the result.

In case (iv), I and III share exactly one null direction, so there are local coordinates for which $\mathrm{I}=2 m d x d y$ and III $=2 F d x d y+G d y^{2}$ with $G \neq 0$. Thus II $=2 M d x d y+N d y^{2}$ with $N \neq 0$, while $\operatorname{Cod}(\mathrm{I}, \mathrm{II})$ is given by

$$
M_{x} / M=m_{x} / m, \quad\left(M_{y}-N_{x}\right) / M=m_{y} / m .
$$

If $H=M / m \equiv c,(61)$ gives $N=N(y)$, so there are new coordinates $x, y$ for which all previous formulas hold with $N= \pm 1$. Since $\tilde{K} \neq 0$, we have $\tilde{H}^{2} \equiv \tilde{K} \neq 0$, so that (59) gives $c \neq 0$ and

$$
\text { III }=2\left(c^{2} m d x d y \pm c d y^{2}\right) .
$$

Thus the Christoffel symbols for III satisfy $\Gamma_{12}^{1} \equiv \Gamma_{12}^{2} \equiv 0$. By Lemma $11^{-}, \nu$ is harmonic on $U$. Conversely, if $\nu$ is harmonic on $U$, the I-null coordinates provided by Lemma 16 give III $=2 F d x d y \pm d y^{2}$, so that $\mathrm{II}=2 M d x d y+N d y^{2}$ with $N=$ $\pm F / 2 M \neq 0$, and $\operatorname{Cod}(\mathrm{I}, \mathrm{II})$ is expressed by (61). The first equation in (61) gives $F M_{x}=F_{x} M$, so $N_{x} \equiv 0$, and the second gives $F M_{y}=F_{y} M$. Thus $H=M / F$ is constant.

In case $(\mathrm{v}), \tilde{K} \equiv \tilde{H} \equiv 0$ gives $K \equiv H \equiv 0$, so $\nu$ is a constant map, and $\nu$ is automatically harmonic on $U$.

In case (vi), $\tilde{K} \equiv 0$ gives $K \equiv 0$, while $\tilde{H} \neq 0$ gives $H \neq 0, \tilde{H}^{\prime}>0$ and $H^{\prime}>0$. There are doubly orthogonal coordinates for the pair I, III so that

$$
\mathrm{I}=l d x^{2}+n d y^{2}, \quad \mathrm{II}=k_{1} l d x^{2}, \quad \mathrm{III}=2 H \mathrm{II}=k_{1}^{2} l d x^{2},
$$

while $\operatorname{Cod}(\mathrm{I}, \mathrm{II})$ is given by

$$
2 l\left(k_{1}\right)_{y}=-l_{y}, \quad 0=n_{x} .
$$

Near any point of $f(U)$, choose coordinates $u^{1}, u^{2}$ so that $u^{1} \equiv x$ and $u^{2} \equiv 0$ on $f(U)$, with the meric $h=h_{i j} d u^{i} d u^{j}$ for $\Sigma^{+}$or $\Sigma^{-}$on $f(U)$ satisfying

$$
h_{11}=k_{1}^{2} l, \quad h_{12} \equiv 0 .
$$

The formula for $(d v)_{i j}^{\gamma}$ in $\$ 3.1$ of [7] together with $m_{x}=0$ from (62) and (63) shows that $\tau \equiv 0$ for $\nu$ if and only if

$$
\left(k_{1}^{2} l\right)_{y}=0, \quad l_{x} / l=\left(k_{1}^{2} l\right)_{x} / k_{1}^{2} l
$$

which simplifies to

$$
\left(k_{1}\right)_{y}\left(1-k_{1}\right)=0, \quad\left(k_{1}\right)_{x}=0,
$$

by (62). If $H=k_{1}$ is constant on $U$, (64) holds, so that $\nu$ is harmonic. Conversely, if $\nu$ is harmonic on $U,(64)$ gives $k_{1}$ locally constant on $U$ wherever $k_{1} \neq 1$. By continuity, $k_{1}=H$ must be constant on the connected set $U$. 
Proof of Theorem 13. The argument is easier than in the previous proof, since $\tilde{H}=H(\mathrm{II}, \mathrm{III})=H(\mathrm{I}, \mathrm{II})=H, \tilde{K}=K(\mathrm{II}, \mathrm{III})=K(\mathrm{I}, \mathrm{II})=K$ and $\tilde{H}^{\prime}=H^{\prime}(\mathrm{II}, \mathrm{III})$ $=H^{\prime}(\mathrm{I}, \mathrm{II})=H^{\prime}$. Here $\mathrm{II}^{\prime}(\mathrm{II}, \mathrm{III})=\mathrm{II}^{\prime}, \mathrm{III}^{\prime}(\mathrm{II}, \mathrm{III})=\mathrm{III}^{\prime}$ and $W(\mathrm{II}, \mathrm{III}) \propto W$. Finally, since $K \neq 0$, only cases (i)-(iv) must be handled. By Fact 3, Cod(I, II) gives Cod(III, II) and we apply Lemmas 4-7 to the pair III, II. Using (6), one proceeds as above. Details are left to the reader.

\section{REFERENCES}

1. J. K. Beem and P. E. Ehrlich, Glohal Lorentzian geometr, Dekker, New York, 1981.

2. L. Bers, Quasiconformal mappings and Teichmüller's theorem (Analytic Functions Conf.). Princeton Univ. Press, Princeton, N.J., 1960, pp. 89-119.

3. E. Calabi, Examples of Bernstein problems for some nonlinear equations, Proc. Sympos. Pure Appl. Math. 15 (1968), 223-230.

4. S. Y. Cheng and S. T. Yau, Maximal spacelike hypersurfaces in the Lorentz-Minkowski spaces, Ann. of Math. (2) 104 (1976), 407-419.

5. S. S. Chern, Geometrical interpretation of the sinh-Gordon equation, Ann. Polon. Math. 39 (1980), $74-80$.

6. S. S. Chern and S. I. Golberg, On the volume decreasing properties of a class of real harmonic mappings, Amer. J. Math. 97 (1975), 133-147.

7. J. Eells and L. Lemaire, $A$ report on harmonic maps, Bull. London Math. Soc. 10 (1978), 1-68.

8. L. Graves and K. Nomizu, On sectional curvature of indefinite metrics, Math. Ann. 232 (1978), $267-272$.

9. C. H. Gu, On the Cauchy problem for harmonic maps defined on two-dimensional Minkowski space, Comm. Pure Appl. Math. 33 (1980), 727-738.

10. D. Hoffman and R. Osserman, The area of the generalized Gaussian image and the stability of minimal surfaces in $S^{n}$ and $R^{n}$, Math. Ann. (to appear).

11. H. Hopf and W. Rinow, Über den Bergriff der vollständigen differential-geometrischen Fläche, Comment. Math. Helv. 3 (1931), 209-225.

12. H. Hopf, Über Flächen mit einer Relation zwischen den Hauptkrummungen, Math. Nachr. 4 (1951), $232-249$.

13. T. Klotz, Some uses of the second conformal structure on strictly convex surfaces, Proc. Amer. Math. Soc. 14 (1963), 1281-1288.

14. Surfaces harmonically immersed in $E^{3}$, Pacific J. Math. 21 (1967), 78-87.

15. T. Klotz and R. Osserman, Complete surfaces in $E^{3}$ with constant mean curvature, Comment. Math.

Helv. 41 (1966-67), 313-318.

16. T. K. Milnor, Efimov's theorem about complete immersed surfaces of negative curvature, Advances in Math. 8 (1972), 474-543.

17. The curvature of $\alpha \mathrm{I}+\beta \mathrm{II}+\gamma \mathrm{III}$ on a surface in a 3-manifold of constant curvature, Michigan Math. J. 22 (1975), 247-255.

18. __ Harmonically immersed surfaces, J. Differential Geom. 14 (1979), 205-214.

19. Codazzi pairs on surfaces, Global Differential Geometry an Global Analysis, Lecture Notes

in Math., vol. 838, Springer-Verlag, Berlin and New York, 1981, 263-273.

20. ___ Abstract Weingarten surfaces, J. Differential Geom. 15 (1980), 365-380.

21. _— Mapping surfaces harmonically into $E^{n}$, Proc. Amer. Math. Soc. 78 (1980), 269-275.

22. __ The energy-1 metric on harmonically immersed surfaces, Michigan Math. J. 28 (1981), $341-346$.

23. __ Classifying harmonic maps of a surface in $E^{n}$, Amer. J. Math. 103 (1981), 211-218.

24. __ Are harmomically immersed surfaces at all like minimally immersed surfaces?, Seminar on Minimal Submanifolds, (E. Bombieri, editor), Ann. of Math. Studies (to appear).

25. Characterizing harmonic immersions of surfaces with indefinite metric, Proc. Nat. Acad. Sci. U.S.A. 79 (1982), 2143-2144.

26. __ Surfaces in Minkowski 3-space on which $H$ and $K$ are linearly related (preprint).

27. C. W. Misner, Harmonic maps as models for physical theories, Phys. Rev. D 18 (1978), 4510-4524.

28. B. O’Neill, Semi-Riemannian geometry, Academic Press, New York, 1982. 
29. K. Pohlmeyer, Integrable Hamiltonian systems and interactions through quadratic constraints, Comm. Math. Phys. 46 (1976), 207-221.

30. P. K. Raschewski, Riemannsche Geometrie und Tensoranalysis, VEB Deutsch. Verlag Wiss., Berlin, 1959.

31. E. H. Ruh and J. Vilms, The tension field of the Gauss map, Trans. Amer. Math. Soc. 149 (1970), 569-573.

32. G. Springer, An introduction to Riemann surfaces, Addison-Wesley, Reading, Mass., 1957.

33. D. J. Struik, Lectures on classical differential geometry, Addison-Wesley, Reading, Mass., 1950.

34. A. E. Treibergs, Entire spacelike hypersurfaces of constant mean curvature in Minkowski space, Invent. Math. (to appear).

35. B. Wegner, Codazzi-tensoren und Kennzeichungen sphärischer immersionen, J. Differential Geom. 9 (1974), 61-70.

36. J. A. Wolf, Surfaces of constant mean curvature, Proc. Amer. Math. Soc. 17 (1966), 1103-1111.

37. , Spaces of constant curvature, 4th ed., Publish or Perish, Berkeley, Calif., 1977.

Department of Mathematics, Hill Center, Rutgers University, New Brunswick, New Jersey 08903 